\title{
Unlocking Structure-Self-Assembly Relationships in Cationic Azobenzene Photosurfactants
}

\author{
Camille Blayo $^{\text {a }}$, Judith E. Houston ${ }^{\text {b }}$, Stephen M. King ${ }^{\mathrm{c}}$ and Rachel C. Evans ${ }^{\mathrm{d}}$ \\ a School of Chemistry and CRANN, University of Dublin, Trinity College, College Green, \\ Dublin 2, Ireland. \\ b Jülich Centre for Neutron Science (JCNS) at Heinz Maier-Leibnitz Zentrum (MLZ), \\ Forschungszentrum Jülich GmbH, Lichtenbergstr. 1, 85748 Garching, Germany. \\ ${ }^{c}$ ISIS Pulsed Neutron Source, STFC, Rutherford Appleton Laboratory, Didcot, Oxfordshire \\ OX110QX, U.K. \\ ${ }^{\mathrm{d}}$ Department of Materials Science \& Metallurgy, University of Cambridge, U.K. \\ Email: rce26@cam.ac.uk
}

\begin{abstract}
Azobenzene photosurfactants are light-responsive amphiphiles that have garnered significant attention for diverse applications including delivery and sorting systems, phase transfer catalysis and foam drainage. The azobenzene chromophore changes both its polarity and conformation (trans-cis isomerization) in response to UV light, while the amphiphilic structure drives self-assembly. Detailed understanding of the inherent relationship between the molecular structure, physicochemical behaviour and micellar arrangement of azobenzene photosurfactants is critical to their usefulness. Here, we investigate the key structure-functionassembly relationships in the popular cationic alkylazobenzene trimethylammonium bromide (AzoTAB) family of photosurfactants. We show that subtle changes in the surfactant structure (alkyl tail, spacer length) can lead to large variations in the critical micelle concentration, particularly in response to light, as determined by surface tensiometry and dynamic light
\end{abstract}


scattering. Small-angle neutron scattering (SANS) studies also reveal the formation of more diverse micellar aggregate structures (ellipsoids, cylinders, spheres) than predicted based on simple packing parameters. The results suggest that while the azobenzene core resides in the effective hydrophobic segment in the trans-isomer, it forms part of the effective hydrophilic segment in the cis-isomer, due to the dramatic conformational and polarity changes induced by photoisomerization. The extent of this shift in the hydrophobic-hydrophilic balance is determined by the separation between the azobenzene core and the polar head group in the molecular structure. Our findings show that judicious design of the AzoTAB structure enables selective tailoring of the surfactant properties in response to light, such that they can be exploited and controlled in a reliable fashion.

\section{KEY WORDS}

Surfactant, azobenzene, light, self-assembly, micelle, small-angle neutron scattering, photoisomerization, critical micelle concentration 


\section{INTRODUCTION}

Stimuli-responsive materials have attracted significant interest due to their potential applications as shape-memory systems, ${ }^{1}$ molecular machines ${ }^{2}$ or artificial muscles. ${ }^{3}$ These materials change their physicochemical properties in response to one or more stimuli in their local environment, such as light, ${ }^{4-5} \mathrm{pH}^{6}$ or heat. ${ }^{7}$ Light is a very effective stimulus as it can be applied selectively at different wavelengths and intensities, with complete temporal and spatial control. ${ }^{5,8}$ The trans-cis photoisomerization of azobenzene in particular, has been extensively exploited in the design of light-responsive materials. ${ }^{9-10}$ Upon irradiation with UV light, photoisomerization leads to dramatic changes in the size, shape and dipole moment of the molecule, which can be reversed through the application of blue light or heat. ${ }^{11}$ In this context, azobenzene photosurfactants, in which the photoresponsive motif is located within a surfactant structure (usually the tail), are particularly attractive due to the combination of surface activity, self-assembly and photoresponse in a single molecule. ${ }^{12}$ These unique properties have led to the investigation of azobenzene photosurfactants in a variety of applications, which have been recently reviewed, ${ }^{13}$ and include microfluidic devices, ${ }^{8}$ cargo delivery ${ }^{14}$ and micellar catalysis. ${ }^{15}$

Like conventional surfactants, when present above a critical concentration in aqueous solution, photosurfactants tend to self-assembly into micelles. ${ }^{12}$ For ionic surfactants in water, the absolute value of the critical micelle concentration (CMC) is largely determined by the length of the hydrophobic segment, which provides the driving force for self-assembly, with long hydrophobic segments leading to low CMCs. ${ }^{16}$ The shape, size and long-range organisation of the self-assembled aggregates are influenced by concentration, temperature and ionic strength of the medium, while the molecular packing within micelles is largely dependent on the shape and volume of individual molecules. ${ }^{17}$ In photosurfactants, the azobenzene group is usually incorporated within the hydrophobic segment of the trans- 
isomer. ${ }^{13}$ However, photoisomerization to the $c i$-isomer induces a significant change in the effective length and volume of the surfactant tail, ${ }^{18}$ and also leads to a large increase in the dipole moment $\left(\mu=3.0 \mathrm{D}\right.$ for the cis-azobenzene core $\left.{ }^{19}\right)$, which makes the molecule significantly more hydrophilic. As such, it can be difficult to distinguish the effective hydrophilic and hydrophobic segments of the molecule, particularly if there is only a small separation between the azobenzene core and the head group. ${ }^{20-21}$ A further complication arises from the fact that photoisomerization never results in complete conversion and instead a photostationary state comprised of an excess of the predominant isomer is obtained. ${ }^{22}$

Given these challenges, it is perhaps not surprising that there have been limited structure-self-assembly studies on azobenzene photosurfactants. ${ }^{23-24}$ The most studied structure is a cationic azobenzene photosurfactant bearing a trimethylammonium bromide (TAB) polar head group, which is separated from the azobenzene (Azo) core by an alkoxy spacer $\left(\mathrm{O}-\mathrm{R}_{2}\right)$. A pendant alkyl tail $\left(\mathrm{R}_{1}\right)$ completes the hydrophobic segment of the surfactant (AzoTAB, Figure 1). AzoTAB surfactants have received significant attention for their potential applications, for example in the design of light-responsive gels ${ }^{25-27}$ and DNA, ${ }^{28-30}$ modified graphene oxide, ${ }^{31-32}$ photomanipulation of droplets $^{8}{ }^{8}$ templating $^{33}$ and photoluminescent complexes. ${ }^{34}$ In contrast, little has been done to determine the relationship between molecular design and function in AzoTABs. Hayashita et al. studied the effect of the length of the hydrophobic segment $\left(\mathrm{R}_{1}+\mathrm{R}_{2}<10\right)$ of AzoTABs in the trans- and cis- forms and determined that an increase in the length of the alkyl tail $\left(\mathrm{R}_{1}\right)$ led to a decrease in both the absolute conductivity and CMC. ${ }^{35}$ Other studies have investigated the interaction of $\mathrm{C}_{4} \mathrm{AzoOR} \mathrm{R}_{2} \mathrm{TAB}$, with different spacer lengths $\left(\mathrm{R}_{2}=6,8,10,12\right)$ with oppositely-charged particles (microgels ${ }^{25}, \mathrm{DNA}^{29}$ ). Elongated spacers were shown to result in more hydrophobic AzoTABs, which facilitated compaction of DNA, while photoisomerization led to the reverse decompaction. ${ }^{36}$ Similarly, Diguet et al. reported that for $\mathrm{C}_{4} \mathrm{OAzoOR} \mathrm{OAB}_{2} \mathrm{TAB}\left(\mathrm{R}_{2}=2,5,8\right)$ 
optimal control of DNA compaction required a compromise in the photosurfactant design, between the best reversibility (short spacer length) and compaction efficiency (long spacer length). ${ }^{30}$ Only a few studies have investigated the size and shape of AzoTAB micelles above the $\mathrm{CMC}$ in detail, with the primary focus centred on the effect of photoisomerization on the general aggregate structure. ${ }^{32,37-38}$

In this study we systematically investigate structure-function-self-assembly relationships in the AzoTAB family at short-to-intermediate chain lengths (up to 14 carbons, excluding the azobenzene core). To achieve this, we have rationally designed a series of structures that enable us to explore and extend the current limits of available AzoTABs in the literature. Our primary focus is to elucidate how the length of the hydrophobic segment, and the relative sizes of the spacer and alkyl tails of which it is composed, influence the surface tension, CMC and micelle shape and size. Small-angle neutron scattering studies are used to examine the effect of concentration, temperature and photoisomerization on the micellar structure. In particular, we investigate how the relative separation between the azobenzene core and the head group (controlled by spacer length) affects the effective head-to-tail volume upon photoisomerization. The application of packing parameters ${ }^{39}$ to predict the aggregate shape for non-conventional surfactants such as AzoTABs will also be examined. Our end goal is to provide key insight into the structural factors controlling surface activity and selfassembly in AzoTAB surfactants, which will enable the strategic design of new structures whose properties are specifically tailed towards their intended application as stimuliresponsive materials. 


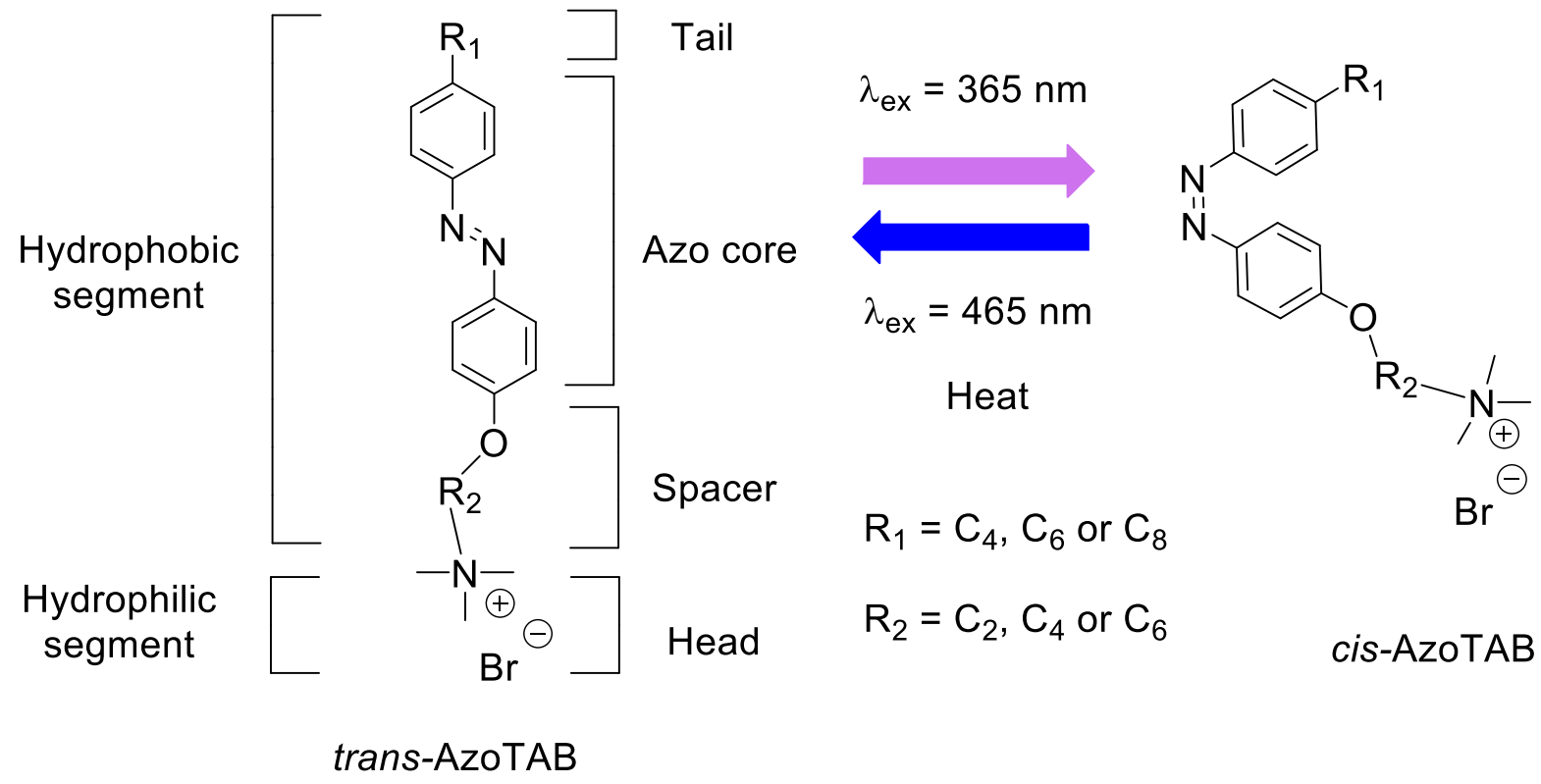

Figure 1. Molecular structure of the AzoTAB photosurfactants investigated in this study. $\mathrm{R}_{1}$ corresponds to the pendant alkyl tail and $\mathrm{R}_{2}$ indicates the spacer. The trans-cis photoisomerization occurs at $\lambda_{\mathrm{ex}}=365 \mathrm{~nm}$ and the reversible cis-trans photoisomerization at $\lambda_{\mathrm{ex}}=465 \mathrm{~nm}$ or with heat.

\section{EXPERIMENTAL}

Materials. AzoTAB photosurfactants were synthesized following a slight modification to a previously reported method. ${ }^{40}$ Full details of the synthetic procedures and associated characterisation can be found in the Supporting Information (SI, Figure S1-15). To the best of our knowledge, $\mathbf{C}_{6} \mathbf{A z o O C}_{4}$ TAB and $\mathbf{C}_{8} \mathbf{A z o O C}_{6}$ TAB are new compounds, while $\mathbf{C}_{4} \mathrm{AzoOC}_{4} \mathbf{T A B},{ }^{32,}, 35 \mathbf{C}_{8} \mathbf{A z o O C}_{2} \mathbf{T A B}^{35}$ and $\mathbf{C}_{4} \mathbf{A z o O C}_{6} \mathbf{T A B}^{41}$ have been synthesized previously.

Photoirradiation Studies. Trans-cis photoisomerization was performed by exposing aqueous solutions $(3 \mathrm{~mL})$ of the photosurfactant in a quartz cuvette $(10 \mathrm{~mm}$ path length) on one side to a UV light-emitting diode (LED) $\left(\right.$ Prolight $^{\circledR}$ ) with an illumination wavelength of $365 \mathrm{~nm}$ and an incident power output of $5 \mathrm{~mW} \mathrm{~cm} \mathrm{~m}^{-2}$ in a custom-built dark box. For all experiments performed in this study, the progress of photoisomerization was followed by UV/Vis absorption spectroscopy. The photostationary state was determined to be reached when the 
absorbance at $315 \mathrm{~nm}$ (cis-isomer) or $350 \mathrm{~nm}$ (trans-isomer) did not increase further upon continued irradiation (Figure S16, SI). The photostationary state was typically obtained after 3 min irradiation for dilute solutions $(<20 \mathrm{mM})$ and was comprised of $\sim 95 \%$ cis- and $\sim 5 \%$ trans-isomers.

Surface Tensiometry (ST). Surface tensiometry measurements were performed on a Kibron EZ-Piplus surface tensiometer using a DyneCups plastic cuvette $(3 \mathrm{~mL})$ and a DyneProbe ring. The instrument uses the Du Noüy ring method to record ST data. The method enables the interfacial force between two media to be recorded. The obtained data were recorded on AquaPiPlus software and were averaged from 5 runs for each sample. Millipore water was used as a reference sample.

Dynamic Light Scattering (DLS). DLS measurements were performed using a Zetasizer Nano series nano-ZS instrument (Malvern Instruments, U.K.). The apparatus is equipped with a He-Ne laser $\left(\lambda_{\mathrm{ex}}=633 \mathrm{~nm}, 4.0 \mathrm{~mW}\right.$ power source $)$. Detection of the scattering intensity was done at a backscattering angle of $173^{\circ}$. Millipore water was filtered 5 times, prior to preparation of the solutions. For each sample, three measurements were performed, each taking an average of 15 scans. Cumulant analysis of the autocorrelation function was used to determinate the polydispersity and the mean hydrodynamic diameter ( $Z$-average) of each sample.

Small-Angle Neutron Scattering (SANS). SANS measurements were performed on the LOQ beamline at the ISIS Pulsed Neutron Source (STFC Rutherford Appleton Laboratory, Didcot, U.K.). ${ }^{42}$ A simultaneous $q$-range of $0.009-0.24 \AA^{-1}$ was achieved using an incident wavelength range of 2.2-10.0 $\AA$ separated by time-of-flight over a sample-detector distance of $4.1 \mathrm{~m}$, where $q=4 \pi \sin (\theta / 2) / \lambda, \lambda$ is the incident wavelength and $\theta$ is the scattering angle. Samples were prepared in $\mathrm{D}_{2} \mathrm{O}$ to ensure good neutron scattering contrast and placed in quartz 
cuvettes (Hellma UK, Type 120, $1 \mathrm{~mm}$ pathlength). Each raw scattering data set was corrected for the detector efficiency, sample transmission and background scattering and converted to scattering cross-section data $\left(\partial \Sigma / \partial \Omega\right.$ vs. q) using the software Mantid. ${ }^{43}$ These data were converted to absolute scattering intensity $\left(\mathrm{cm}^{-1}\right)$ by comparison with the scattering from a standard sample (a solid blend of hydrogenated and perdeuterated polystyrene) in accordance with established procedures. ${ }^{44}$ SANS profiles were then fitted using to Sphere, ${ }^{45}$ oblate Ellipsoid ${ }^{46}$ or Elliptical Cylinder models ${ }^{46}$ including polydispersity as described in the text using nonlinear least-squares optimization with the SasView program (version 4.0.1). ${ }^{47}$ Full details of the models can be found in the SI. Validation of the model fits was achieved using the theoretical mass of dry material $\left(\varphi_{\text {dry }}\right)$ that the fit returned (see section 5.2.1 of the S.I. for full details).

Cryogenic Scanning Emission Microscopy (Cryo-SEM). Cryo-SEM measurements were performed using a Zeiss-Ultra Scanning Electron Microscope at an accelerating voltage of 2-5 $\mathrm{kV}$ using an In-lens detector. Samples were plunged into sub-cooled liquid $\mathrm{N}_{2}$ to freeze and then transferred in-vacuo to the cold stage of the SEM cryo-preparation chamber.

\section{RESULTS}

\section{Design of AzoT ABs}

AzoTAB structures were designed to explore a range of intermediate lengths $(\mathrm{C} 8, \mathrm{C} 10, \mathrm{C} 14)$ for the hydrophobic segment, which comprises the Azo core, a moderate length alkyl tail ( $\mathrm{R}_{1}$ $=4,6$ or 8$)$ and a short to moderate length spacer $\left(R_{2}=2,4\right.$ or 6$)$. The relative lengths of the spacer and the alkyl chain control the extent at which the Azo group is embedded in the hydrophobic segment. These AzoTABs were chosen to explore molecular dimensions that supplement previously reported structures. ${ }^{48-49}$ Detailed sample compositions are shown in Table 1. The samples are denoted as $\mathbf{C}_{x} \mathbf{A z o O C} \mathbf{C}_{y} \mathbf{T A B}$, where $x$ corresponds to the number of 
carbon atoms in the alkyl tail and $y$ corresponds to the number of carbon atoms in the spacer.

$\mathrm{C}_{8} \mathrm{AzoOC}_{2} \mathrm{TAB}$ and $\mathrm{C}_{4} \mathrm{AzoOC}_{6} \mathrm{TAB}$ have been reported previously, but a detailed investigation of the surface activity and micellar structure was not performed. ${ }^{29}, 35$ $\mathrm{C}_{4} \mathrm{AzoOC}_{\mathbf{4}}$ TAB is the most extensively studied AzoTAB structure $^{32,35}$ and is included as a benchmark for the other compounds.

The self-assembly of conventional surfactants above the CMC can be reasonably approximated from the packing parameter, $P$, which is given by $P=V / a l$, where $V$ is the volume of the hydrophobic tail of the surfactant, $l$ is the tail length and $a$ is the effective head group area. ${ }^{39}$ The packing parameter predicts the shape and size of micellar aggregates based on geometrical considerations. ${ }^{50}$ Calculations of $V$ and $l$ for conventional surfactants are usually given by the Tanford equations ${ }^{51}$ and, for a given $a$, the $V / l$ ratio remains constant, independent of the hydrophobic tail length. ${ }^{17}$ The calculated packing parameters for the transand cis-isomers of the AzoTABs are shown in Table 1 (see Table S4 in the SI for calculation of $P$ ). Similar to the analogous cationic alkyltrimethylammonium bromide surfactants with no Azo core, ${ }^{52}$ spherical micelles are expected for all investigated AzoTABs, in both isomeric forms. However, while the packing parameters for the trans- and cis-isomers appear to be independent of the molecular structure, these estimates do not consider the effect of the position of the azobenzene core within the photosurfactant. In particular, short spacers and long alkyl chains are expected to significantly affect the hydrophobicity of AzoTABs, leading to stark changes in the physicochemical properties upon photoisomerization of the azobenzene core. ${ }^{35}$

Table 1. Molecular structure and calculated packing parameters, $P$, of the AzoTABs studied. $\mathrm{R}_{1}+\mathrm{R}_{2}$ corresponds to the total length of the hydrophobic segment (excluding the Azo core), varying from short $\left(\mathrm{R}_{1}+\right.$ $\left.\mathrm{R}_{2}=8\right)$ to intermediate $\left(\mathrm{R}_{1}+\mathrm{R}_{2}=14\right)$. From the calculated $P$ values, all AzoTABs (in both isomeric forms) are expected to form spherical micelles $(0.286<P<0.335){ }^{53}$

$\begin{array}{llllll}\text { AzoTAB } & \mathbf{R}_{1} & \mathbf{R}_{2} & \mathbf{R}_{1}+\mathbf{R}_{2} & \boldsymbol{P}_{\text {trans }} & \boldsymbol{P}_{\text {cis }}\end{array}$

(Tail) 


\begin{tabular}{|c|c|c|c|c|c|}
\hline \multicolumn{6}{|c|}{ (Spacer) } \\
\hline $\mathrm{C}_{4} \mathrm{AzoOC}_{4} \mathrm{TAB}$ & 4 & 4 & 8 & 0.29 & 0.34 \\
\hline $\mathrm{C}_{4} \mathrm{AzoOC}_{6} \mathrm{TAB}$ & 4 & 6 & 10 & 0.29 & 0.34 \\
\hline $\mathrm{C}_{6} \mathrm{AzoOC}_{4} \mathrm{TAB}$ & 6 & 4 & 10 & 0.29 & 0.34 \\
\hline $\mathrm{C}_{8} \mathrm{AzoOC}_{2} \mathrm{TAB}$ & 8 & 2 & 10 & 0.29 & 0.34 \\
\hline $\mathrm{C}_{8} \mathrm{AzoOC}_{6} \mathrm{TAB}$ & 8 & 6 & 14 & 0.30 & 0.34 \\
\hline
\end{tabular}

\section{Surface Tension, Solubility and the CMC.}

The CMCs of AzoTABs in water $\left(20^{\circ} \mathrm{C}\right)$ were determined for both isomers using surface tensiometry and dynamic light scattering. As a representative example, Figure 2a shows the variation in surface tension as a function of concentration for $\mathbf{C}_{4} \mathbf{A z o} \mathbf{O C} \mathbf{C}_{6} \mathbf{T A B}$. The CMC is reached when the surface tension becomes independent of surfactant concentration, ${ }^{54}$ at $0.40 \mathrm{mM}$ and $1.05 \mathrm{mM}$ for the trans- and cis-forms, respectively. The CMC was confirmed by DLS, whereby the intensity of scattering light increases significantly upon the onset of micelle formation (Figure 2b). The CMCs determined for all AzoTABs by both ST and DLS are summarized in Table 2. It is worth noting that the Krafft temperature of alkyltrimethylammonium bromide surfactants (e.g. $\left.\mathrm{C}_{16} \mathrm{TAB}\right)$ is known to be around $25^{\circ} \mathrm{C}$ in water. ${ }^{55}$ Interestingly, among the AzoTABs studied here, only $\mathbf{C}_{6} \mathbf{A z o O C}_{\mathbf{4}} \mathbf{T A B}$ displays low solubility in water at $20{ }^{\circ} \mathrm{C}$, and flocculates, suggesting that this temperature is below the Krafft point. ${ }^{56}$ Controlled heating of $\mathbf{C}_{6} \mathbf{A z o O C}_{4} \mathbf{T A B}$ (5 $\mathrm{mM}$ in water) confirmed the Krafft temperature to be $27^{\circ} \mathrm{C}$. The values for the CMC obtained are in good agreement with those previously reported for AzoTAB surfactants. ${ }^{29,32,35}$ The absolute values of the surface tension isotherm above the $\mathrm{CMC}$ are in good agreement with reported values for azobenzene photosurfactants, ${ }^{32}$ with the longest hydrophobic segment showing the lowest surface tension (i.e. $\mathrm{R}_{1}+\mathrm{R}_{2}=14$ ). 

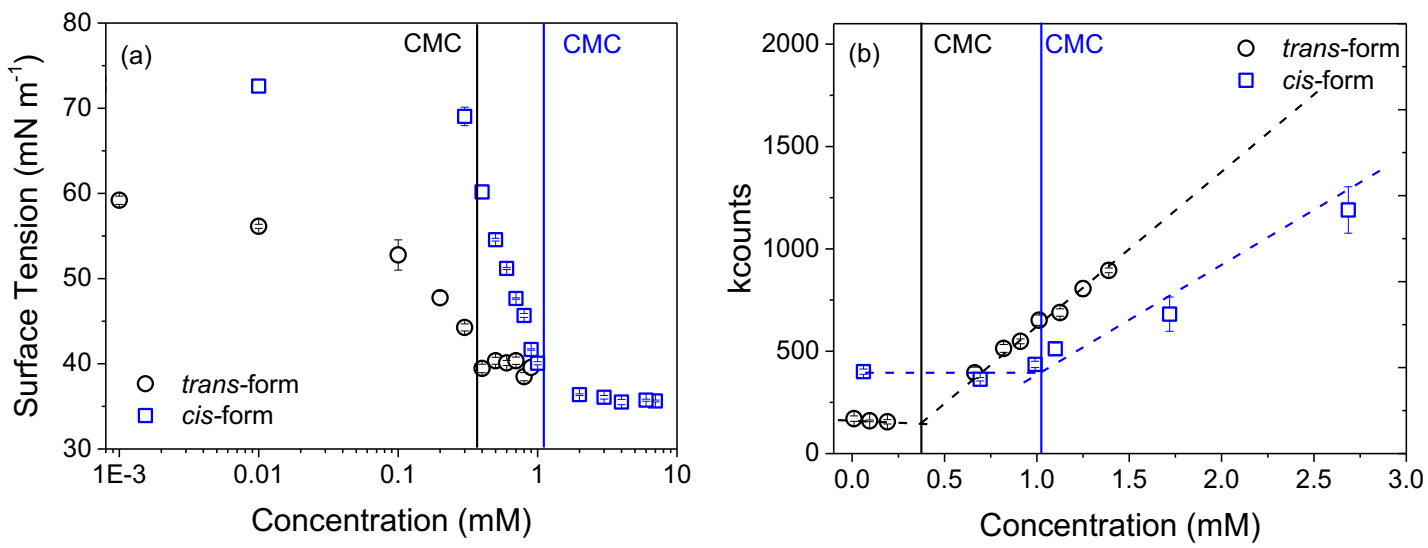

Figure 2. Determination of the $\mathrm{CMC}$ of $\mathbf{C}_{4} \mathbf{A z o O C} \mathbf{C}_{6} \mathbf{T A B}$ in water at $20{ }^{\circ} \mathrm{C}$. Variation of (a) surface tension and (b) light scattering as a function of concentration. The data points show the trend before (trans-form, black circles) and after irradiation with UV light at $\lambda_{\mathrm{ex}}=365 \mathrm{~nm}$ for $5 \mathrm{~min}$ (predominantly cis-form, blue squares). The $\mathrm{CMC}$ values are obtained from the intersection between the two trends (solid lines). The error bars show the standard deviation of the mean values obtained over 5 (ST) or 3 (DLS) measurements. The dashed lines serve only to guide the eye.

Table 2. Summary of the CMCs obtained for AzoTABs by surface tensiometry (ST) and dynamic light scattering (DLS) at $20^{\circ} \mathrm{C}$.

\begin{tabular}{|c|c|c|c|c|c|}
\hline \multirow{2}{*}{ AzoTAB } & \multicolumn{2}{|c|}{ trans-CMC (mM) } & \multicolumn{2}{|c|}{ cis-CMC (mM) } & \multirow{2}{*}{$\begin{array}{c}\text { trans-/cis-CMC (mM) } \\
\text { Literature }\end{array}$} \\
\hline & ST & DLS & ST & DLS & \\
\hline $\mathrm{C}_{4} \mathrm{AzoOC}_{4} \mathrm{TAB}$ & $1.10 \pm 0.02$ & $1.2 \pm 0.1$ & $2.50 \pm 0.02$ & $2.7 \pm 0.2$ & $1.2 / 2.7^{b} ; 1.0 / 2.0^{c}$ \\
\hline $\mathrm{C}_{4} \mathrm{AzoOC}_{6} \mathrm{TAB}$ & $0.40 \pm 0.02$ & $0.4 \pm 0.1$ & $1.05 \pm 0.02$ & $1.1 \pm 0.1$ & $\sim 0.5 / \mathrm{NR}^{d}$ \\
\hline $\mathbf{C}_{6} \mathbf{A z o O C}_{4} \mathrm{TAB}^{a}$ & $0.19 \pm 0.03$ & $0.2 \pm 0.1$ & $0.62 \pm 0.03$ & $0.7 \pm 0.1$ & \\
\hline $\mathrm{C}_{8} \mathrm{AzoOC}_{2} \mathrm{TAB}$ & $0.34 \pm 0.02$ & $0.3 \pm 0.1$ & $0.82 \pm 0.03$ & $0.8 \pm 0.1$ & $0.3 / 0.8^{b}$ \\
\hline $\mathrm{C}_{8} \mathrm{AzoOC}_{6} \mathrm{TAB}$ & $0.09 \pm 0.02$ & $0.1 \pm 0.1$ & $0.15 \pm 0.03$ & $0.2 \pm 0.1$ & \\
\hline
\end{tabular}

${ }^{a}$ Corresponds to the critical aggregation concentration at $25{ }^{\circ} \mathrm{C} .{ }^{b}$ From Hayashita et al. ${ }^{35}$ - determined by conductivity $\left(\mathrm{T}=25^{\circ} \mathrm{C}\right) .{ }^{c}$ From McCoy et al. ${ }^{32}$ - determined by pendant drop tensiometry $\left(\mathrm{T}=\right.$ not specified). ${ }^{d}$ From Zakrevskyy et al. ${ }^{29}$ - determined by isothermal titration calorimetry $\left(\mathrm{T}=25^{\circ} \mathrm{C}\right)$, cis-isomer not reported (NR).

Three key trends can be identified upon comparison of the CMCs of the AzoTABs studied here with those previously reported as a function of the alkyl tail length $\left(R_{1}\right)$ and the spacer length $\left(\mathrm{R}_{2}\right)$ for both the trans- and cis-isomers. For a given surfactant, the CMC of the cis-isomer is always higher than that of the trans-isomer, which can be rationalized based on the increased dipole moment, and thus hydrophilicity, upon photoisomerization. Secondly, the hydrophobic driving force for micellization is driven by the total length of the hydrophobic 
segment of the structure, with a larger length (i.e. $\mathrm{R}_{1}+\mathrm{R}_{2}$ ) leading to a lower CMC. This has been previously demonstrated for short hydrophobic segments $\left(R_{1}+R_{2} \leq 8\right)^{35}$ and is confirmed here for much longer hydrophobic segments (up to $R_{1}+R_{2}=14$ ), enabling the CMCs to be tuned from $\mathrm{mM}$ to $\mu \mathrm{M}$. Finally, for a fixed hydrophobic segment length, a longer alkyl tail (and thus a shorter spacer) leads to a smaller CMC, for example $\mathbf{C}_{4} \mathbf{A z o O C}_{6} \mathbf{T A B}>$ $\mathbf{C}_{8} \mathbf{A z o O C}_{6}$ TAB (Table 2 and Figure S17-20, SI). Similarly, for a fixed alkyl chain length, the CMC decreases as the spacer length increases.

The difference in $\mathrm{CMC}(\triangle \mathrm{CMC})$ for the cis- and trans-isomers as a function of the total number of carbons in the hydrophobic segment $\left(\mathrm{R}_{1}+\mathrm{R}_{2}\right)$ is shown in Figure 3 . The $\triangle \mathrm{CMC}$ is an important parameter as it represents the range of concentrations in which micelles exist in the trans-form only but are expected to return to unimers upon photoisomerization to the cis-isomer. This property has previously been exploited for the reversible encapsulation and release of small molecules, for example for catalysis ${ }^{57}$ or drug release. ${ }^{58}$ There is an apparent sweet spot for obtaining the maximum $\triangle \mathrm{CMC}$, which occurs for a total hydrophobic segment length of six carbons, irrespective of the tail and spacer lengths from which the segment is composed. As the total hydrophobic segment length is increased or decreased, the magnitude of the $\triangle \mathrm{CMC}$ decreases significantly. If we consider the extreme cases, i.e. a very short or very long hydrophobic segment, the low $\triangle \mathrm{CMC}$ can be rationalized. For short hydrophobic segments, the Azo core dominates both the length and the volume, and the difference between the hydrophobic driving force for micellization (dominated by the alkyl tail) for the two isomers is small. Similarly, for long hydrophobic segments, the longer alkyl tail and/or spacer increases the flexibility of the hydrophobic segment in both isomers, leading to an increase in the free volume irrespective of photoisomerization. For a hydrophobic segment length of $\mathrm{R}_{1}+\mathrm{R}_{2}=6$, there is an apparent compromise between these two factors, whereby the free volume of the hydrophobic segment, 
and therefore packing of the molecules in the micelle, is controlled predominantly by the photoisomerization of the Azo core.

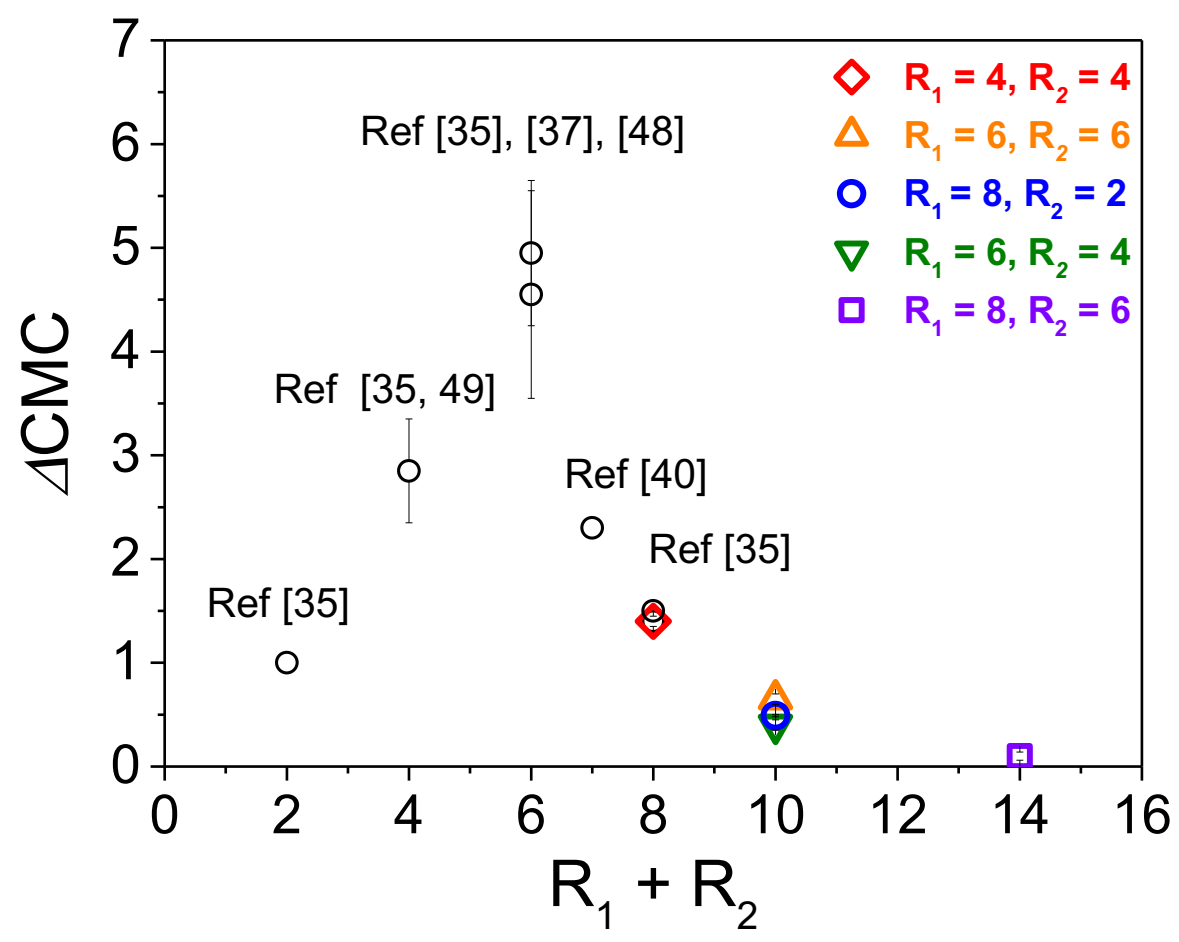

Figure 3. Comparison of the difference in CMC $(\triangle \mathrm{CMC})$ for cis- and trans-isomers of AzoTABs as a function of the total number of carbons in the hydrophobic segment $\left(\mathrm{R}_{1}+\mathrm{R}_{2}\right)$. Data are presented from this study (coloured circles) and previous reports (black circles). The errors bars result from different reported CMC in the literature.

Analysis of the absolute values reveals that an apparently larger $\triangle \mathrm{CMC}$ is obtained for a longer spacer length, i.e. $\mathbf{C}_{2} \mathbf{A z o O C}_{4} \mathbf{T A B}>\mathbf{C}_{4} \mathbf{A z o O C} \mathbf{C}_{2} \mathbf{T A B}$. If we consider the homologous series of $\left(R_{1}+R_{2}=10\right)$ AzoTABs, the same trend is observed, with the absolute value of $\triangle \mathrm{CMC}$ increasing with the spacer length, i.e. $\mathbf{C}_{4} \mathbf{A z o O C}_{6} \mathbf{T A B}>\mathbf{C}_{8} \mathbf{A z o O C}_{2} \mathbf{T A B}$. This suggests that the spacer length contributes a more important role to the effect that photoisomerization has on the packing of the molecules within the micelles. The spacer separates the hydrophilic head group from the Azo core, which is nominally hydrophobic in the trans-form. However, the increase in the dipole moment upon photoisomerization renders the Azo core more hydrophilic. As such, for short spacer lengths, the separation between the 
polar head group and the Azo core is small, which could indicate that photoisomerization leads to an increase in the effective head group area of the molecule to include the now polar Azo core.

\section{Above the CMC: Size and Shape of AzoTAB Micelles}

Effect of concentration. SANS experiments were carried out to investigate the size and shape of AzoTAB micelles formed above the $\mathrm{CMC}$ as a function of the molecular structure. Figure 4 presents the SANS profiles for the AzoTABs in $\mathrm{D}_{2} \mathrm{O}$ as a function of concentration $(\mathrm{T}=20$

$\left.{ }^{\circ} \mathrm{C}\right)$. The SANS window for these experiments is calculated to be 10 to $1000 \AA(d=2 \pi / q)$, which is suitable for the observation of micellar aggregates $(\sim 30 \AA) .{ }^{59}$ Scaling concepts were initially used to assess the data in the intermediate $q$-regime $\left(q=0.03-0.01 \AA^{-1}\right)$. For $\mathrm{C}_{4} \mathrm{AzoOC}_{4} \mathrm{TAB}, \mathrm{C}_{4} \mathrm{AzoOC}_{6} \mathrm{TAB}$ and $\mathrm{C}_{8} \mathrm{AzoOC}_{2} \mathrm{TAB}$ at $5 \mathrm{mM}$ (Figure 4a-c, red squares), the SANS data scale as $\sim q^{-4}$ and level off as a Guinier plateau at low- $q\left(q<0.02 \AA^{-1}\right)$, which is consistent with the formation of spherical-like aggregates. ${ }^{60}$ Fitting of these SANS data to an oblate Ellipsoid model ${ }^{46}$ yielded a short equatorial radius $(R)$ of 25, 25 and $20 \AA$ and a long equatorial radius $(L)$ of 39,45 and $52 \AA$ for $\mathbf{C}_{4} \mathbf{A z o O C}_{4} \mathbf{T A B}, \mathbf{C}_{4} \mathbf{A z o O C}_{6} \mathbf{T A B}$ and $\mathbf{C}_{8} \mathbf{A z o O C} \mathbf{C}_{2} \mathbf{T A B}$, respectively (Table 3 ). The representative structure of an oblate ellipsoid is shown in Figure 4f. These values are in excellent agreement with values reported for both conventional cationic alkylammonium bromide surfactant solutions such as $\mathrm{C}_{16} \mathrm{TAB}$ above

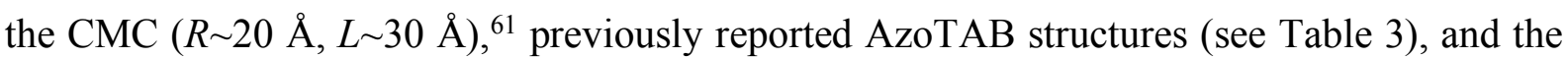
estimated lengths of the hydrophobic segment (Table S4). The fits were validated using Guinier analysis ${ }^{62}$ to determine the radius of gyration $\left(R_{\mathrm{g}}\right)$, which yielded comparable values (see Table S2 and Figure S21, SI). It appears from the fitted parameters that the short radius, $R$, is not affected by the length of the hydrophobic segment or by the position of the azobenzene core within it, and remains around 20-25 $\AA$. In contrast, the long equatorial 
radius, $L$, increases with the length of the hydrophobic segment, which suggests that micellar growth occurs mainly in this direction.

The SANS data of $\mathbf{C}_{\mathbf{8}} \mathbf{A z o O} \mathbf{C C}_{\mathbf{6}} \mathbf{T A B}$ in $\mathrm{D}_{2} \mathrm{O}$ at $5 \mathrm{mM}$ (Figure $4 \mathrm{~d}$ ) decay as $\sim q^{-3.2}$ over a large $q$ window $\left(0.05-0.1 \AA^{-1}\right)$ and tend towards a Guinier plateau at low- $q$, which suggests the formation of more elongated micelles. Fitting of the data to the oblate Ellipsoid Model ${ }^{46}$ leads to radii of $R=28 \AA$ and $L=88 \AA$. Despite the significant differences in the visual appearance of the SANS profiles, the $R$ and $L$ radii follow the trend in the dimensions of $\mathbf{C}_{4} \mathbf{A z o}_{4} \mathbf{T A B}$, $\mathbf{C}_{4} \mathbf{A z o O C}_{6} \mathbf{T A B}$ and $\mathbf{C}_{8} \mathbf{A z o O C}_{2}$ TAB described above and confirm that micelles grow preferentially in the $L$ direction. Additionally, the eccentricity of the aggregates formed, defined as $\varepsilon=\left(1-R^{2} / L^{2}\right)^{0.5}$ suggests the formation of very flat ellipsoids $(\varepsilon=0.95)$. Finally, the SANS profile of $\mathbf{C}_{6} \mathbf{A z o O C} \mathbf{C}_{4} \mathbf{T A B}$ in $\mathrm{D}_{2} \mathrm{O}$ at $5 \mathrm{mM}$ decays as $\sim q^{-0.8}$ in the intermediate $q$ region (Fig 4e), which suggests the formation of more cylindrical structures. Fitting of the scattering profile to the Elliptical Cylinder model ${ }^{46}$ leads to radii $R$ of $28 \AA$ and $L$ of $49 \AA$, with a height of $H \approx 364 \AA$. Although, this SANS profile differs from the other AzoTABs discussed above, it has been shown that cylindrical structures are spontaneously obtained for cationic CTAB surfactants with $c a .16$ carbons in the hydrophobic tail in salt-free conditions. ${ }^{63} \mathbf{C}_{6} \mathbf{A z o O C}_{4} \mathbf{T A B}$ exhibits comparable $R$ and $L$ radii as the other AzoTABs with a hydrophobic segment length of $\mathrm{C} 10$ (i.e. $\mathbf{C}_{4} \mathbf{A z o O C} \mathbf{C}_{6} \mathbf{T A B}$ and $\mathbf{C}_{8} \mathbf{A z o O C}_{2} \mathbf{T A B}$ ). For all AzoTABs studied, the aggregation number, $N_{\mathrm{agg}}$, determined from the ratio of the volume of dry aggregates to the hydrophobic volume of one unimer (see SI) was found to increase with the total length of the hydrophobic segment (Table 3). Specifically, aggregates with a longer alkyl tail $\left(\mathrm{R}_{1}\right)$ contain more unimers per micelle than AzoTABs with a small $\mathrm{R}_{1}$. This follows the conclusions drawn above for the trend in the $\mathrm{CMC}$, where $\mathrm{R}_{1}$ drives the formation of micelles for the trans-isomers of AzoTABs. 

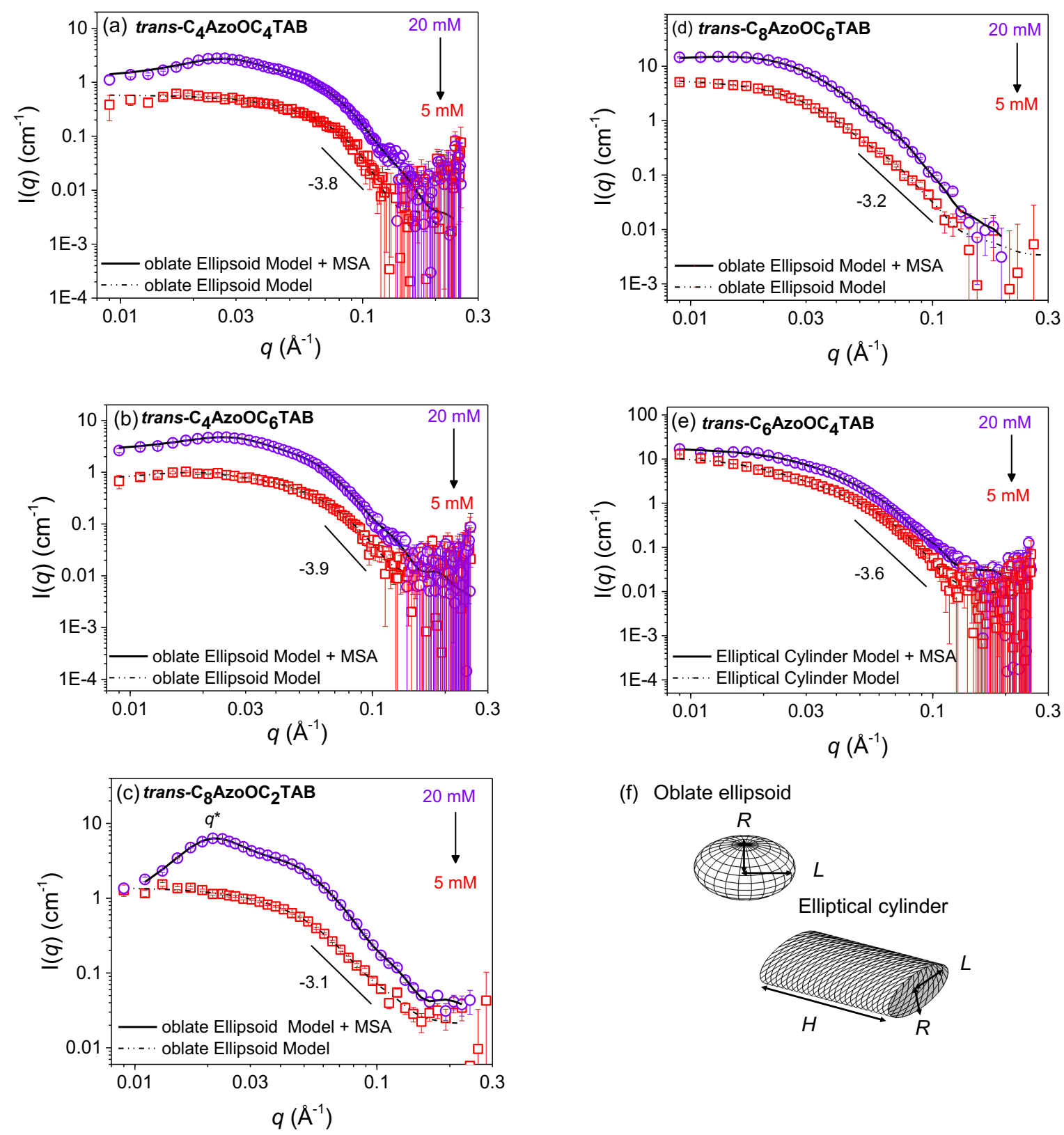

(f) Oblate ellipsoid
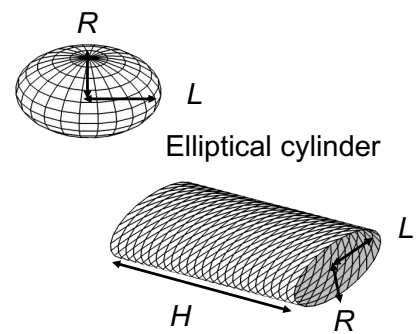

Figure 4. Effect of concentration on the SANS profiles of the trans-isomers of AzoTABs in $\mathrm{D}_{2} \mathrm{O}$ at $20{ }^{\circ} \mathrm{C}$. (a) $\mathrm{C}_{4} \mathrm{AzoOC}_{4} \mathrm{TAB}$, (b) $\mathrm{C}_{4} \mathrm{AzoOC}_{6} \mathrm{TAB}$, (c) $\mathrm{C}_{8} \mathrm{AzoOC}_{2} \mathrm{TAB}$, (d) $\mathrm{C}_{8} \mathrm{AzoOC}_{6} \mathrm{TAB}$ and (e) $\mathrm{C}_{6} \mathrm{AzoC}_{4} \mathrm{TAB}$ in $\mathrm{D}_{2} \mathrm{O}$ at $20 \mathrm{mM}$ (purple circles) and $5 \mathrm{mM}$ (red squares). The black lines correspond to fits of the oblate Ellipsoid model or the Elliptical cylinder model as described in the text. At $20 \mathrm{mM}$, the Hayter-Penfold (MSA) structure factor is included in the fits. (f) Representative structures of oblate ellipsoids and elliptical cylinders, with $R$ and $L$ being the polar and equatorial radii, respectively, and $H$ the length of the cylinder.

The SANS profiles show significant changes upon increasing the concentration to 20 mM. At low- $q$ a Guinier plateau remains for $\mathbf{C}_{4} \mathbf{A z o O C}_{4} \mathbf{T A B}, \mathbf{C}_{4} \mathbf{A z o O C}_{6} \mathbf{T A B}$ and $\mathbf{C}_{8} \mathbf{A z o O C}_{2} \mathbf{T A B}$, but a shoulder appears at $q^{*} \approx 0.03 \AA^{-1}$, which is attributed to repulsive interactions between the positively-charged micelles. To account for this, a Hayter-Penfold 
(MSA) structure factor ${ }^{64}$ was coupled with the appropriate form factor to model these profiles (see SI for further details). The obtained radii upon fitting to the oblate Ellipsoid model confirm that with increasing AzoTAB concentration, micellar growth proceeds along the $L$ axis. Moreover, for a fixed alkyl tail length, this effect becomes more pronounced with an increase in the spacer length, such that for $\mathbf{C}_{4} \mathbf{A z o O C}_{2} \mathbf{T A B}^{37}, \mathbf{C}_{4} \mathbf{A z o O C}_{4} \mathbf{T A B}$ and $\mathbf{C}_{4} \mathbf{A z o O C} \mathbf{6}$ TAB, $L$ values of $39 \AA, 41 \AA$ and $50 \AA$ are obtained, respectively.

For a fixed hydrophobic segment length $\left(\mathrm{R}_{1}+\mathrm{R}_{2}=10\right)$, further differences in the micellar growth are observed. While $\mathbf{C}_{\mathbf{8}} \mathbf{A z o O} \mathbf{C C}_{2} \mathbf{T A B}$ (Figure $4 \mathrm{c}$ ) forms the most elongated micelles at $5 \mathrm{mM}$, further micellar growth is not observed upon increasing the concentration to $20 \mathrm{mM}$, and the micelles appear to become less stretched (decrease in $\varepsilon$ from 0.92 to 0.89 ). This is accompanied by an increase in the scattering intensity at $q^{*}$, indicating an increase in the number of interparticle interactions and thus that the formation of new micelles is favoured over growth. In contrast, $\mathbf{C}_{4} \mathbf{A z o O} \mathbf{C}_{6} \mathbf{T A B}$ micelles (Figure $4 \mathrm{~b}$ ) grow preferentially along the $L$ axis with increasing concentration and by $20 \mathrm{mM}$ reach the same size as $\mathbf{C}_{8} \mathbf{A z o O C}_{2} \mathbf{T A B}$. At $5 \mathrm{mM}, \mathbf{C}_{6} \mathbf{A z o O C}_{4} \mathbf{T A B}$ forms cylindrical micelles, however with increasing concentration, the micellar parameters obtained from a fit to this model change significantly, suggesting growth along the $L$ axis, which is accompanied by a significant decrease in the height of the cylinder from $H=363 \AA$ to $H=162 \AA$. This suggests that a transition from a cylindrical to ellipsoidal structure may occur at higher concentrations, which is supported by a good fit of the data to the oblate Ellipsoid model (see Figure S23, SI). Finally, $\mathbf{C}_{\mathbf{8}} \mathbf{A z o O C} \mathbf{6} \mathbf{T A B}$ shows similar behaviour to $\mathbf{C}_{\mathbf{8}} \mathbf{A z o O C}_{\mathbf{2}} \mathbf{T A B}$, with no significant micellar growth or change in the micelle shape observed upon increasing the concentration. This suggests that the length of the alkyl tail determines both the size and the growth of the micelles formed. 
Table 3. Summary of the parameters obtained for model fitting to the SANS profiles of trans-AzoTABs. For the structures investigated here, the fitted parameters are obtained as described in the text. All samples are in $\mathrm{D}_{2} \mathrm{O}$ at $20{ }^{\circ} \mathrm{C}$. Representative data from the literature are also included for comparison. $\varepsilon$ refers to the eccentricity of the ellipsoidal structure only. $q^{*}$ refer to the interference maximum where interparticle interactions become more significant and $N_{\text {agg }}$ is the aggregation number for the micelle. A polydispersity of 0.1 was applied to all polar and equatorial radii to account for a polydisperse micellar population.

\begin{tabular}{|c|c|c|c|c|c|c|c|c|}
\hline trans-AzoTAB & $\begin{array}{l}\text { Conc. } \\
(\mathrm{mM})\end{array}$ & $\begin{array}{c}\text { Form } \\
\text { factor } P(q)\end{array}$ & $\begin{array}{c}R \\
(\AA)\end{array}$ & $\begin{array}{c}L \\
(\AA)\end{array}$ & $\begin{array}{c}H \\
(\AA)\end{array}$ & $\varepsilon$ & $\begin{array}{c}q^{*} \\
\left(\AA^{-1}\right)\end{array}$ & $N_{\text {agg }}$ \\
\hline $\mathrm{C}_{4} \mathrm{AzoOC}_{2} \mathrm{TAB}^{a}$ & 10 & Ellipsoid & $\approx 21$ & $\approx 39$ & - & - & - & - \\
\hline $\mathrm{C}_{4} \mathrm{AzoOC}_{4} \mathrm{TAB}^{b}$ & 5 & Ellipsoid & $\approx 18$ & $\approx 40$ & - & - & - & - \\
\hline $\mathrm{C}_{4} \mathrm{AzoOC}_{4} \mathrm{TAB}$ & $\begin{array}{c}5 \\
20\end{array}$ & $\begin{array}{l}\text { Oblate } \\
\text { Ellipsoid }^{c}\end{array}$ & $\begin{array}{l}25 \pm 3 \\
24 \pm 1\end{array}$ & $\begin{array}{l}39 \pm 2 \\
41 \pm 1\end{array}$ & - & $\begin{array}{l}0.77 \\
0.81\end{array}$ & $\begin{array}{c}- \\
0.025\end{array}$ & $\begin{array}{c}90 \pm 14 \\
165 \pm 10\end{array}$ \\
\hline $\mathrm{C}_{4} \mathrm{AzoOC}_{6} \mathrm{TAB}$ & $\begin{array}{c}5 \\
20\end{array}$ & $\begin{array}{l}\text { Oblate } \\
\text { Ellipsoid }^{c}\end{array}$ & $\begin{array}{l}25 \pm 1 \\
26 \pm 1\end{array}$ & $\begin{array}{l}45 \pm 1 \\
50 \pm 1\end{array}$ & - & $\begin{array}{l}0.83 \\
0.85\end{array}$ & $\begin{array}{c}- \\
0.024\end{array}$ & $\begin{array}{l}119 \pm 13 \\
255 \pm 24\end{array}$ \\
\hline $\mathrm{C}_{8} \mathrm{AzoOC}_{2} \mathrm{TAB}$ & $\begin{array}{c}5 \\
20\end{array}$ & $\begin{array}{l}\text { Oblate } \\
\text { Ellipsoid }^{c}\end{array}$ & $\begin{array}{l}20 \pm 1 \\
24 \pm 1\end{array}$ & $\begin{array}{l}52 \pm 1 \\
53 \pm 1\end{array}$ & - & $\begin{array}{l}0.92 \\
0.89\end{array}$ & $\begin{array}{c}- \\
0.021\end{array}$ & $\begin{array}{l}209 \pm 21 \\
309 \pm 22\end{array}$ \\
\hline $\mathrm{C}_{8} \mathrm{AzoOC}_{6} \mathrm{TAB}$ & $\begin{array}{c}5 \\
20\end{array}$ & $\begin{array}{l}\text { Oblate } \\
\text { Ellipsoid }^{c}\end{array}$ & $\begin{array}{l}28 \pm 1 \\
29 \pm 1\end{array}$ & $\begin{array}{l}88 \pm 1 \\
87 \pm 1\end{array}$ & - & $\begin{array}{l}0.95 \\
0.94\end{array}$ & $\begin{array}{c}- \\
0.022\end{array}$ & $\begin{array}{l}669 \pm 30 \\
753 \pm 27\end{array}$ \\
\hline $\mathrm{C}_{6} \mathrm{AzoOC}_{4} \mathrm{TAB}$ & $\begin{array}{c}5 \\
20\end{array}$ & $\begin{array}{l}\text { Elliptical } \\
\text { Cylinder }^{c}\end{array}$ & $\begin{array}{l}28 \pm 1 \\
27 \pm 1\end{array}$ & $\begin{array}{l}49 \pm 1 \\
53 \pm 1\end{array}$ & $\begin{array}{c}364 \pm 11 \\
162 \pm 2\end{array}$ & $\begin{array}{l}0.82 \\
0.86\end{array}$ & $\begin{array}{c}- \\
0.019\end{array}$ & - \\
\hline
\end{tabular}

${ }^{a}$ From Akamatsu et $a l .{ }^{37 b}$ From McCoy et $a l .{ }^{32}{ }^{c}$ Hayter-Penfold structure factor for electrostatic interactions included in this fit as described in the text.

For all AzoTABs, the micellar structures become significantly drier upon increasing the surfactant concentration, irrespective of the shape, which appears to reach a limit $20-25 \%$, and has been assumed to be concentrated around the polar head region (Table S2). ${ }^{65-66}$ The decrease in the water content is consistent with increased packing between molecules as indicated by the increase in the aggregation number. $\mathbf{C}_{6} \mathbf{A z o} \mathbf{O}_{4} \mathbf{T A B}$ is the only exception, forming significantly more wet aggregates at $5 \mathrm{mM}\left(\mathrm{X}_{\text {Solv }}=62 \%\right)$, which decreases to $\mathrm{X}_{\text {Solv }}=$ $36 \%$ at $20 \mathrm{mM}$, which is consistent with the different aggregate shape formed for this AzoTAB.

Effect of Photoisomerization. Figures $5 \mathrm{a}$ and $5 \mathrm{~b}$ show the SANS profiles of $\mathrm{C}_{4} \mathrm{AzoOC}_{4} \mathrm{TAB}$ and $\mathbf{C}_{4} \mathbf{A z o O C} \mathbf{C}_{6}$ TAB in $\mathrm{D}_{2} \mathrm{O}(20 \mathrm{mM})$ before and after irradiation at $365 \mathrm{~nm}$. The scattering profiles of the $c i s$-isomers are shifted towards high $q$, with $q^{*}$ from $q=0.025$ to $0.035 \AA^{-1}$ and 
$q=0.023$ to $0.039 \AA^{-1}$, for $\mathbf{C}_{4} \mathbf{A z o O C}_{4} \mathbf{T A B}$ and $\mathbf{C}_{4} \mathbf{A z o O} \mathbf{C}_{6} \mathbf{T A B}$ respectively, which indicate that micelles are more closely packed following photoisomerization. A Guinier plateau remains visible at low $q$ followed by an abrupt decay which scales as $\sim q^{-4.5}$. For $\mathbf{C}_{4} \mathbf{A z o O C}_{4} \mathbf{T A B}$, fitting of the SANS data to the Sphere model ${ }^{45}$ gave smaller, wet micelles (58\% solvent) with a radius $R$ of $26 \AA$ and a low aggregation number $\left(N_{\text {agg }}=42\right)$. Increasing the spacer length by 2 carbons, whilst keeping the same alkyl chain $\mathrm{R}_{1}\left(\mathbf{C}_{4} \mathbf{A z o} \mathbf{O C}_{6} \mathbf{T A B}\right)$, leads to dryer (35\%) and slightly larger micelles $\left(R=26 \AA, N_{\text {agg }}=67\right)$. The SANS profile of cis- $\mathbf{C}_{\mathbf{8}} \mathbf{A z o O C} \mathbf{C}_{\mathbf{2}} \mathbf{T A B}$ can also be fitted with the Sphere model, leading to small $(R=25 \AA)$ and dry (17\%) aggregates. The Hayter-Penfold structure factor is required to produce good fits to the data, due to the large $q^{*}$ at $0.038 \AA^{-1}$, which leads to a decrease in the estimated interparticle distance from $299 \AA$ to $165 \AA$ (Figure 5c). The shift in $q^{*}$ upon photoisomerization is the highest of all the AzoTABs studied here, suggesting a very closely packed environment. For the same hydrophobic segment length, the calculated radii follow the order $\mathbf{C}_{4} \mathrm{AzoOC}_{6} \mathrm{TAB}>\mathrm{C}_{4} \mathrm{AzoOC}_{4} \mathrm{TAB}>\mathbf{C}_{8} \mathbf{A z o O C}_{2} \mathbf{T A B}$, where this time the spacer length seems to control the size of the micelle.

Figure 5d shows the SANS data and fits for trans- and cis- $\mathbf{C}_{8} \mathbf{A z o O} \mathbf{C C}_{6} \mathbf{T A B}$ in $\mathrm{D}_{2} \mathrm{O}$, in which a large difference in the scattering profiles of the two isomers is observed. After photoisomerization, a slight upturn is observed at $q=0.03 \AA^{-1}$ followed by a sharp decay of $\sim q^{-3.9}$. While the scattering data for both isomers are best described by the oblate Ellipsoid model $^{46}$ coupled with the Hayter-Penfold structure factor, there are stark differences between the obtained fitting parameters. The oblate ellipsoidal micelles formed by the cis-isomer are much smaller $(R=23 \AA, L=36 \AA)$ and less flat than the trans counterparts $(R=29 \AA, L=87$ $\AA$ ), with an eccentricity of 0.77 , indicating that the packing of unimers along the $L$ axis is less favourable in the cis-micelles. Finally, the SANS profile of $c i s-\mathbf{C}_{6} \mathbf{A z o O C} \mathbf{C}_{4} \mathbf{T A B}$ is very similar to that of the trans-form and could also be fit to the Elliptical Cylinder model, ${ }^{46}$ to 
give $R=22 \AA, L=46 \AA$ and $H=260 \AA$, which are in good agreement with the fitting parameters for the trans-isomer (Figure 5e). As the presence of the cis-isomer was confirmed optically by UV/Vis absorption spectroscopy, before and after the SANS measurements (Figure S24), it appears that the bent cis-form can be accommodated quite effectively within the elliptical cylinder structure, with minimal changes to the structural parameters. It is worth noting here that complete conversion to the cis-isomer is never achieved, with a small fraction of trans-isomer ( $\sim 5 \%)$ remaining in solution due to the photostationary state (Figure S15).

For all samples, the fitting parameters were validated by model-independent analysis of the radius of gyration (Figure S21, Table S3). The SANS data for the cis-isomers of all the AzoTABs in $\mathrm{D}_{2} \mathrm{O}(5 \mathrm{mM})$ were also collected and fitted to the same models used for the corresponding sample at $20 \mathrm{mM}$ (see Table 4 for obtained fitting parameters). As expected, at the lower concentration, the obtained micelles were smaller and wetter with a lower $N_{\text {agg. }}$.

Table 4. Summary of the parameters obtained for model fitting to the SANS profiles for cis-AzoTABs. For the structures investigated here, the fitted parameters are obtained as described in the text. All samples are in $\mathrm{D}_{2} \mathrm{O}$ at $20{ }^{\circ} \mathrm{C}$. Representative data from the literature are also included for comparison. $\varepsilon$ refers to the eccentricity of the ellipsoidal structure only. $q^{*}$ references to the interference maximum where interparticle interactions become more significant and $N_{\text {agg }}$ is the aggregation number for the micelle. A polydispersity of 0.1 was applied to all polar and equatorial radii to account for a polydisperse micellar population.

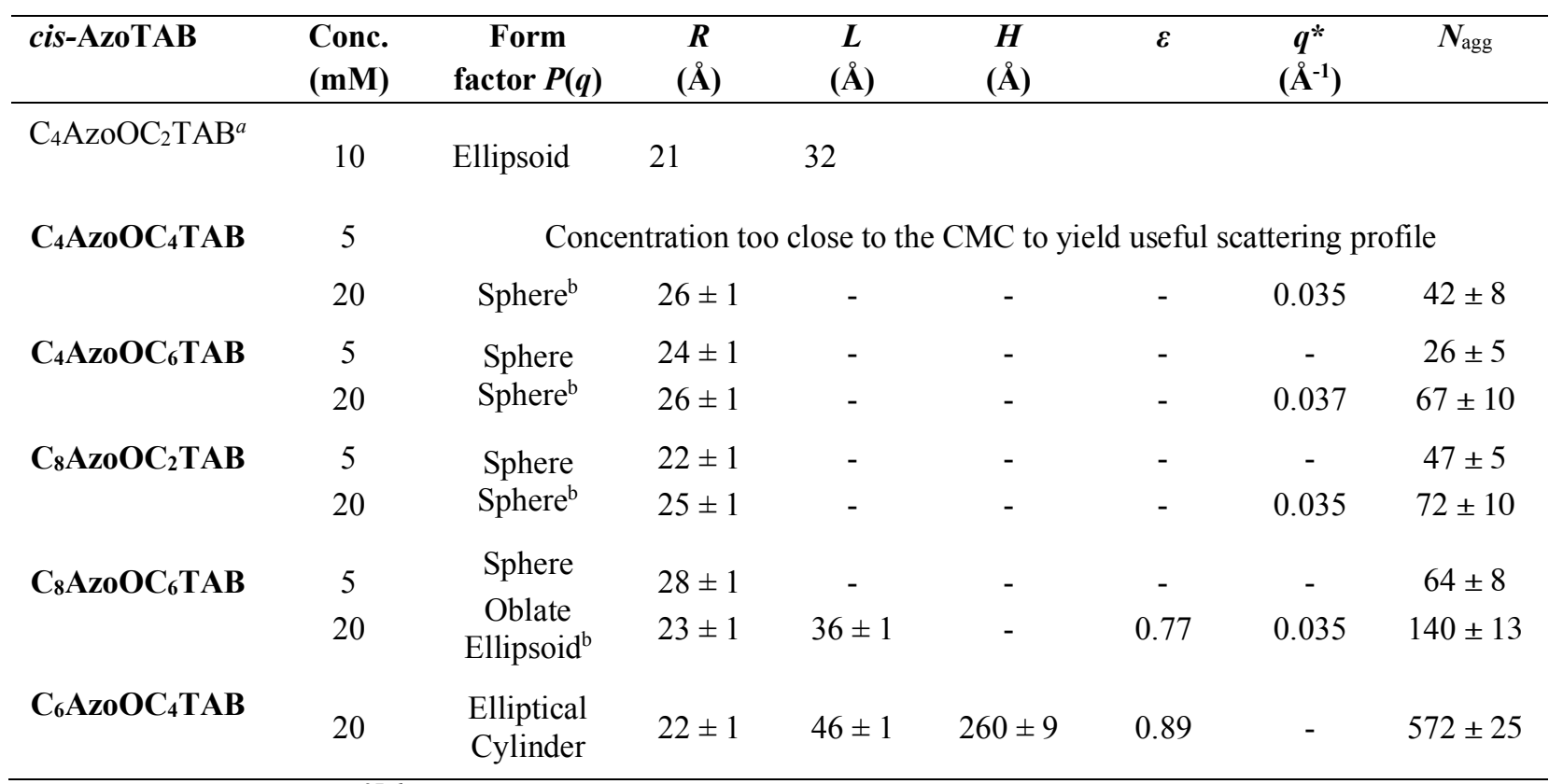

${ }^{a}$ From Akamatsu et al. ${ }^{37 b}$ Hayter-Penfold structure factor for electrostatic interactions included in this fit as described in the text. 

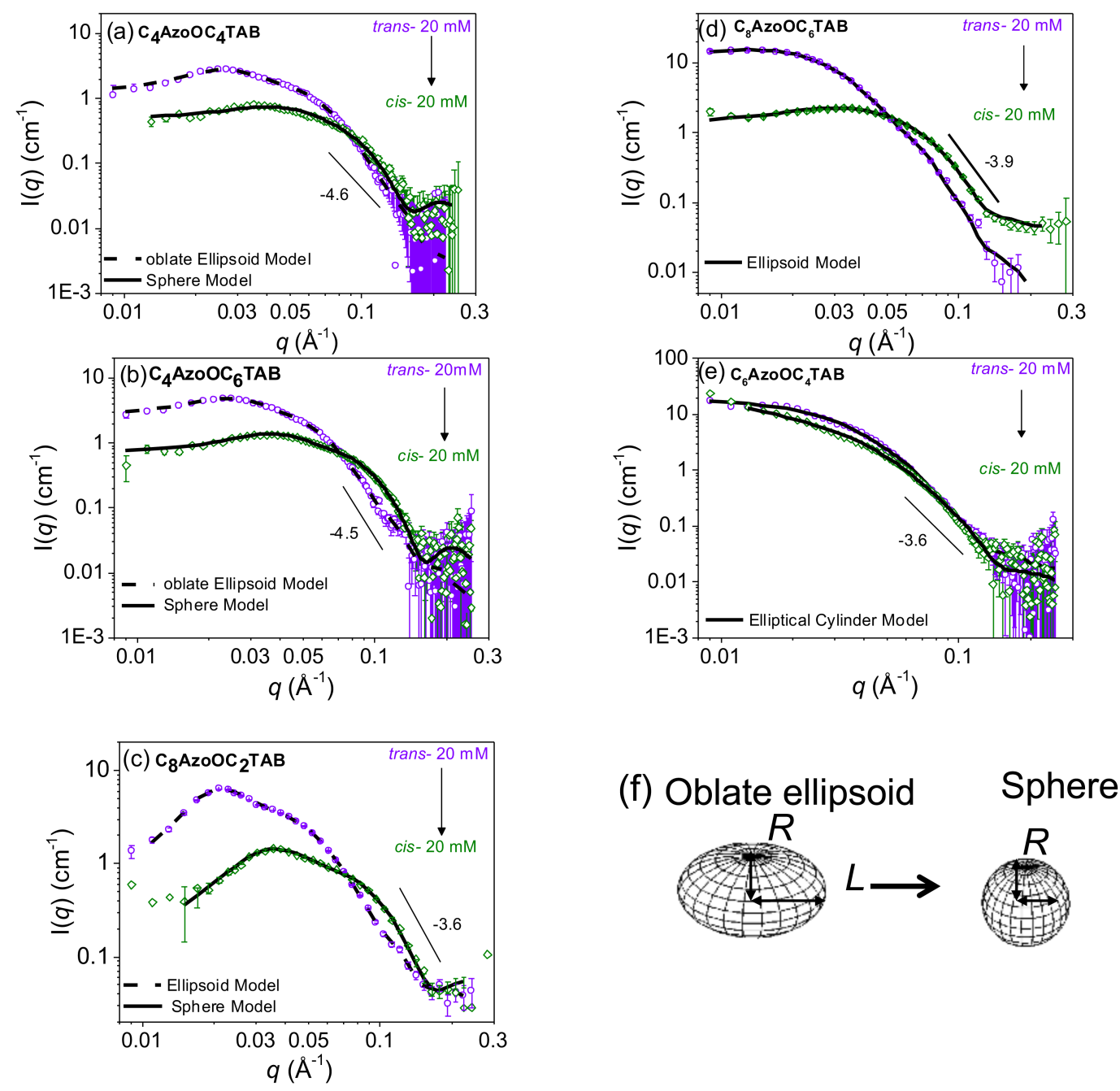

Figure 5. Effect of photoisomerization on the SANS profiles of AzoTABs in $\mathrm{D}_{2} \mathrm{O}\left(20 \mathrm{mM}, 20^{\circ} \mathrm{C}\right)$. Scattering data presented for the trans- (purple circles) and cis-isomers (green diamonds) of (a) $\mathbf{C}_{4} \mathbf{A z o O C}_{4} \mathbf{T A B}$, (b) $\mathrm{C}_{4} \mathrm{AzoOC}_{6} \mathrm{TAB}$, (c) $\mathbf{C}_{8} \mathrm{AzoOC}_{2} \mathrm{TAB}$, (d) $\mathbf{C}_{8} \mathrm{AzoOC}_{6} \mathrm{TAB}$ and (e) $\mathrm{C}_{6} \mathrm{AzoOC}_{4} \mathrm{TAB}$. The solid and dashed black lines correspond to fits to the data as described in the text. (f) A model of the ellipse to sphere transition of AzoTAB micelles upon photoisomerization, where $R$ is the polar radius and $L$ is the equatorial radius.

\section{Visualization of the Micelle Structure}

Cryo-SEM measurements were performed on the images of the trans- and cis-isomers of $\mathbf{C}_{\mathbf{8}} \mathbf{A z o O C}_{2} \mathbf{T A B}(20 \mathrm{mM})$. This AzoTAB was selected based on the large difference in the SANS scattering profiles observed upon photoisomerization. Figure 6 shows the cryo-SEM images of the trans- and cis-isomers of $\mathbf{C}_{\mathbf{8}} \mathbf{A z o O C} \mathbf{C}_{2} \mathbf{T A B}$. Image analysis revealed the presence of a primary population of aggregates with diameters of $7.5 \pm 1.0 \mathrm{~nm}$ and $4 \pm 0.5 \mathrm{~nm}$ for the trans- and cis-isomers, respectively (see Figure S26, SI). In addition, several very 
large aggregates are observed, $\sim 200 \mathrm{~nm}$ in diameter, which are believed to be artefacts of the sample preparation process (e.g. particle agglomeration and/or crystallisation of solid surfactant). Upon increasing magnification, the larger trans-aggregates appear to be populated with smaller aggregates on the surface (Figure 6b), while cis-form micelles are much smoother (Figure 6d). Increased magnification did not allow the smaller aggregates to be examined in detail, however they seem to match the sizes and shapes determined from the analysis of the SANS data. Finally, the trans-aggregates are isolated, while cis-aggregates are more closely packed, and in some cases lead to the formation of fused micelles (Figure 6c, green circle in Figure 6d). These observations agree with the SANS data, which show a shorter distance between aggregates upon photoisomerization. However, it cannot be excluded that these differences arise due to the stark contrast in the local environment of the micelles in the two techniques (liquid for SANS, cryogenic freezing and freeze-fracture for SEM).
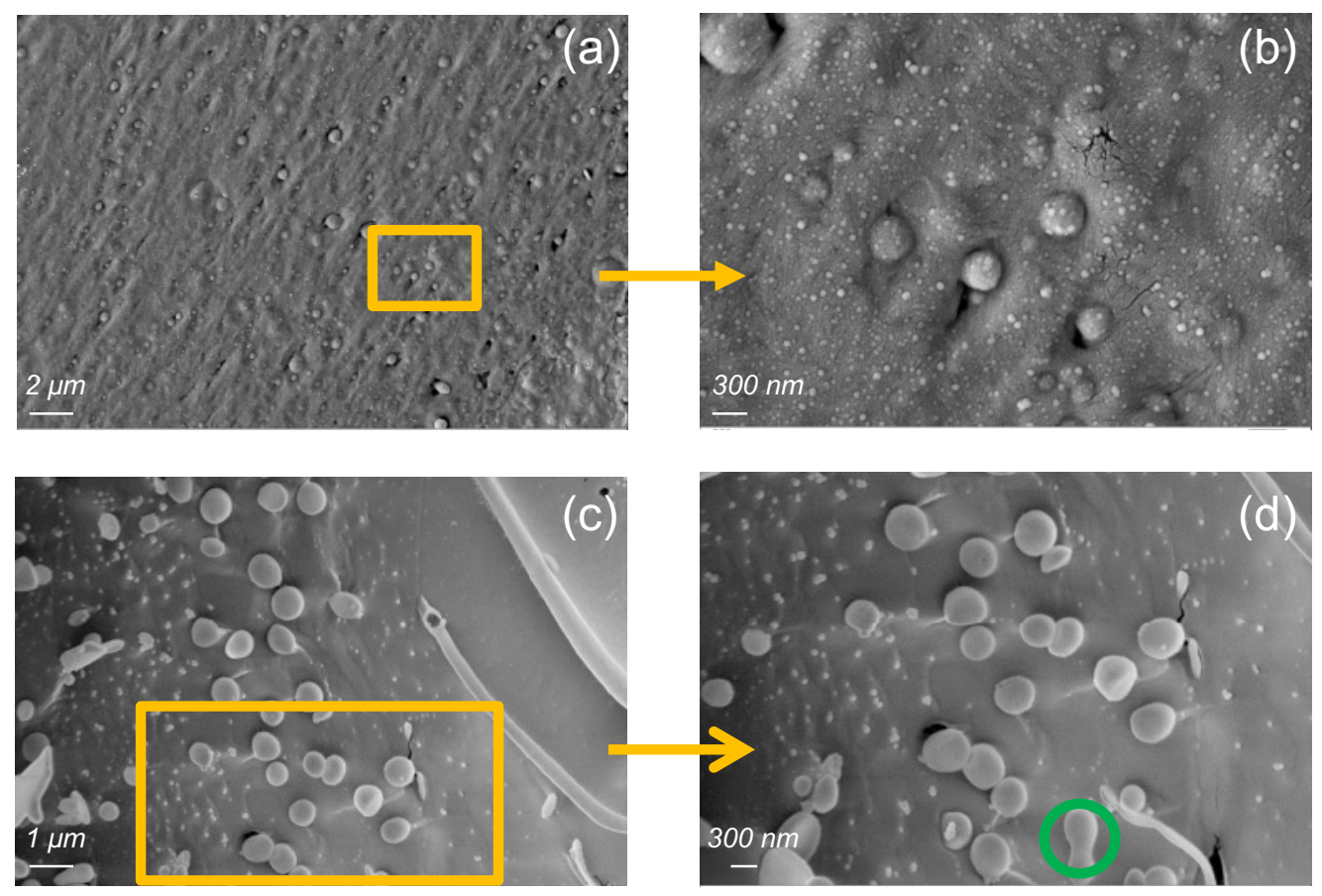

Figure 6. Examination of the aggregate structure of AzoTABs above the CMC (20 mM). Cryo-SEM images of (a), (b) trans- $\mathbf{C}_{8} \mathbf{A z o O C}_{2} \mathbf{T A B}$ and (c), (d) cis- $\mathbf{C}_{8} \mathbf{A z o O C}_{2} \mathbf{T A B}$. Images (b) and (d) are magnified from the orange 
boxes in images (a), (c) and (e), respectively. The green circle in (d) highlights the fusing of two aggregates. Scale bars have been added manually to the images for clarity and the image footers have been removed.

\section{DISCUSSION}

The surface activity and self-assembly characteristics of AzoTABs are controlled by subtle differences in the molecular structure. For trans-isomers, the total length of the hydrophobic segment, which comprises the alkyl tail, the Azo core and the spacer, determines the CMC and size of the micelles formed. For the cis-form, the composition of the hydrophobic segment is less clear cut. Photoisomerization to the cis-isomer leads to a decrease in the volume of the azobenzene core, which is accompanied by a significant increase in the dipole moment from 0 to 3 D. ${ }^{19}$ This results in a significant hydrophilic component within the assumed hydrophobic tail. If the spacer length is reasonably short, photoisomerization will bring the cis-azobenzene core into closer proximity with the polar trimethylammonium bromide head group. In this conformation, it may be more intuitive to consider the polar Azo core and the short spacer as part of the hydrophilic head group, rather than the hydrophobic segment. If this is the case, it may be interesting to consider the maximum spacer length to which this behaviour occurs.

Like conventional surfactants, the value of the CMC is primarily determined by the length of the alkyl chain, with long chains leading to a lower CMC. However, the effect of the molecular structure on the $\triangle \mathrm{CMC}$ for the cis- and trans-isomers is less obvious. There is an apparent sweet spot in the length of the assumed hydrophobic segment for $\mathrm{R}_{1}+\mathrm{R}_{2}=6$, which leads to the maximum difference in the CMCs between the two isomers. Previous SANS studies have shown that like most of the AzoTABs studied here, $\mathrm{C}_{4} \mathrm{AzoOC} \mathrm{C}_{2} \mathrm{TAB}$ also forms ellipsoidal (trans) or pseudo-spherical (cis) in $\mathrm{D}_{2} \mathrm{O}$ (up to $50 \mathrm{mM}$ ). ${ }^{37}$ The large difference in the $\triangle \mathrm{CMC}$ is therefore unlikely to be due to the shape of the aggregate formed. For a given hydrophobic segment length $\left(\mathrm{R}_{1}+\mathrm{R}_{2}=6\right.$ or 10$)$, the largest $\triangle \mathrm{CMC}$ is always obtained for the 
homologue with the longest spacer. To achieve a large $\triangle \mathrm{CMC}$, either the absolute value of the $\mathrm{CMC}_{\text {trans }}$ must decrease, or the $\mathrm{CMC}_{\text {cis }}$ must increase, or a combination of the two must occur. For a given homologous series, increasing the length of the spacer results in a corresponding decrease in the alkyl tail, which leads to an increase in the $\mathrm{CMC}$. The $\mathrm{CMC}_{\text {trans }}$ therefore increases with the spacer length. The large $\triangle \mathrm{CMC}$ must therefore arise from an increase in the $\mathrm{CMC}_{\text {cis. }}$ One possible explanation for this is that upon photoisomerization, the Azo core and spacer now form part of the effective hydrophilic segment of the molecule, and as such the increasing spacer length leads to a larger hydrophilic volume and a higher CMC. The interesting question is if there is a limit on the spacer length at which this transition to a large effective hydrophilic segment occurs and whether the same behaviour would be observed for spacers with an odd number of carbon atoms.

The shapes of the micelles obtained for the fitting of the SANS data to standard geometrical models are in good agreement with the shapes predicted based on the molecular structure using the theoretical packing parameter. With the exception of $\mathbf{C}_{6} \mathbf{A z o O C}_{4} \mathbf{T A B}$, all AzoTABs studied here form ellipsoidal micelles in the trans-form and spherical micelles in the cis-form. For the trans-isomers, micellar growth occurs predominantly in the equatorial axis $(L)$ and there is an apparent maximum micelle size for a given hydrophobic segment length $\left(\mathrm{C}_{10}: L \approx 50 \AA\right)$, above which the formation of new aggregates is favoured over further growth. At a given concentration, the corresponding spherical cis-aggregates are wetter and have a lower $N_{\text {agg. }}$ This is both consistent with a decrease in the molecular packing density within the aggregates due to the "kink" in the Azo core upon photoisomerization and also increased solvation of the effective hydrophilic segment, which based on the arguments above, is now larger in size.

In contrast to the other AzoTABs studied, $\mathbf{C}_{6} \mathbf{A z o O C}_{4} \mathbf{T A B}$ favours the formation of elliptical cylinders in $\mathrm{D}_{2} \mathrm{O}$ in both the trans- and cis-forms, which is in contradiction to the 
theoretical packing parameters for this molecule that predict ellipsoidal/spherical aggregates. However, for the cis-isomer in particular, severe estimates are made for the volume of the azobenzene core. Moreover, the head group area is limited to the cationic terminal group in these calculations, which may not be an accurate reflection of the true effective hydrophilic segment. Nevertheless, there is an apparent preference for $\mathbf{C}_{6} \mathbf{A z o O C} \mathbf{C}_{4} \mathbf{T A B}$ to form cylindrical micelles with a lower curvature and photoisomerization has a limited effect on the shape and size of the aggregates formed. It is as yet unclear which exact feature of the molecular structure drives this preference.

Finally, as noted earlier, photoisomerization does not lead to the formation of a $100 \%$ cis-population, and approximately $5 \%$ of the photostationary state is composed of transisomers. It is interesting to consider if discrete all-trans and all-cis aggregates form under these conditions. Cryo-SEM analysis of $c i s-\mathbf{C}_{8} \mathbf{A z o O C} \mathbf{C}_{2} \mathbf{T A B}$ revealed that spherical aggregates comprise the primary aggregate population in this sample, however isolated ellipsoidal aggregates more characteristic of the trans-isomer were also observed. However, further work is required to further understand if this behaviour is true of the entire sample and also of all AzoTABs.

\section{CONCLUSIONS}

In summary, we have investigated the structure-function-self-assembly characteristics of AzoTAB photosurfactants, with a specific focus on the length and composition of the hydrophobic segment. Our studies, and comparison with the properties of known AzoTABs from the literature have revealed that modest changes in the molecular structure can lead to large variations in the $\mathrm{CMC}$, particularly between the two isomeric forms, and also unexpected aggregate structures. Although these effects are not trivial to predict, subtle trends related to the absolute length of the alkyl tail and the spacer group have been identified. 
Furthermore, it is apparent that photoisomerization leads to a dramatic increase in the hydrophilicity of the molecule, leading to higher CMCs and the formation of smaller and wetter aggregates. Our studies suggest that following photoisomerization, the hydrophobic segment is confined primarily to the alkyl tail, and that the azobenzene core and the spacer form part of the effective hydrophilic segment of the molecule. Further work is now required to investigate if this behavior is restricted to short spacer length, or whether this can be extended to much longer spacer lengths, which should lead to a dramatic shift in the hydrophobic-hydrophilic balance in the trans and cis isomers.

Our findings show that careful design of AzoTAB photosurfactants is needed to tune both the CMC and the nanoscale structure of the resultant micelles. This is of particular importance when selecting the correct surfactant structure for a specific application. For example, variation of the position of the azobenzene core within the hydrophobic segment enables the $\triangle \mathrm{CMC}$ to be subtly optimised and clear alkyl chain/spacer combinations to maximize this property have been identified. The $\triangle \mathrm{CMC}$ concept has been used recently for the recovery of carbon nanotubes ${ }^{32}$ or solubilization of ethylbenzene ${ }^{37}$ in aqueous media, using short AzoTABs (e.g. $\mathrm{C}_{4} \mathrm{AzoOC}_{2} \mathrm{TAB}$ and $\mathrm{C}_{4} \mathrm{AzoOC} \mathrm{C}_{4} \mathrm{TAB}$, respectively) which leads to a large $\triangle \mathrm{CMC}$, but also requires a large amount of material. Conversely, an extended AzoTAB leads to a small $\triangle \mathrm{CMC}$ but reduces the amount of material required. A large $\triangle \mathrm{CMC}$ is desirable for the application of AzoTABs in micellar catalysis, as it may facilitate the recovery of product by simple UV light illumination ${ }^{57}$ and leads to more flexibility in the design of the reaction. This property can be associated with the size and shape of micelles for the design of optimised light-activated nanocarriers for product release.

\section{ASSOCIATED CONTENT}

\section{Supporting Information}


The Supporting Information is available free of charge on the ACS Publications website.

Materials; synthesis and structural characterization of AzoTABs; UV/Vis absorption spectroscopy of AzoTABs; determination of the CMC (ST and DLS data); small-angle neutron scattering; model dependant and independent analysis; procedure to validate SANS model fits; calculation of the packing parameter of AzoTABs.

\section{AUTHOR INFORMATION}

\section{Corresponding Author}

*E-mail: rce26@cam.ca.uk. Tel:+44 1223334356.

\section{Notes}

The authors declare no competing financial interest.

\section{ACKNOWLEDGMENTS}

We thank Dr Clodagh Dooley for assistance with the cryo-SEM. CB thanks Trinity College Dublin for the award of a postgraduate studentship. Experiments at the ISIS Pulsed Neutron and Muon Source were supported by a beamtime allocation from the Science and Technology Facilities Council (Experiment: RB1610028, DOI: $\underline{10.5286 / \text { ISIS.E.79106883 }}$ and 10.5286/ISIS.E.82352186). This work benefited from the use of the SasView application, originally developed under NSF award DMR-0520547. SasView also contains code developed with funding from the European Union's Horizon 2020 research and innovation programme under the SINE2020 project, grant agreement No 654000.

\section{REFERENCES}

(1) Mao, Y.; Ding, Z.; Yuan, C.; Ai, S.; Isakov, M.; Wu, J.; Wang, T.; Dunn, M. L.; Qi, H. J. 3D Printed Reversible Shape Changing Components with Stimuli Responsive Materials. Sci. Rep. 2016, 6, 24761. (2) Angelos, S.; Johansson, E.; Stoddart, J. F.; Zink, J. I. Mesostructured silica supports for functional materials and molecular machines. Adv. Funct. Mater. 2007, 17, 2261-2271.

(3) Islam, M. R.; Li, X.; Smyth, K.; Serpe, M. J. Polymer-Based Muscle Expansion and Contraction. Angew. Chem. Int. Ed. 2013, 52, 10330-10333.

(4) Angelos, S.; Choi, E.; Vögtle, F.; De Cola, L.; Zink, J. I. Photo-Driven Expulsion of Molecules from Mesostructured Silica Nanoparticles. J. Phys. Chem. C 2007, 111, 6589-6592. 
(5) Nguyen, T. D.; Leung, K. C. F.; Liong, M.; Liu, Y.; Stoddart, J. F.; Zink, J. I. Versatile Supramolecular Nanovalves Reconfigured for Light Activation. Adv. Funct. Mater. 2007, 17, 2101-2110.

(6) Nguyen, T. D.; Leung, K. C. F.; Liong, M.; Pentecost, C. D.; Stoddart, J. F.; Zink, J. I. Construction of a pH-Driven Supramolecular Nanovalve. Org. Lett. 2006, 8, 3363-3366.

(7) Lu, Y.; Sun, W.; Gu, Z. Stimuli-responsive nanomaterials for therapeutic protein delivery. J. Control. Release 2014, 194, 1-19.

(8) Diguet, A.; Guillermic, R. M.; Magome, N.; Saint-Jalmes, A.; Chen, Y.; Yoshikawa, K.; Baigl, D. Photomanipulation of a droplet by the chromocapillary effect. Angew. Chem. 2009, 121, 9445-9448.

(9) Kumar, G. S.; Neckers, D. C. Photochemistry of azobenzene-containing polymers. Chem. Rev. 1989, 89, 1915-1925.

(10) Zhao, Y.; Ikeda, T. Smart light-responsive materials: azobenzene-containing polymers and liquid crystals; John Wiley \& Sons: Hoboken, New Jersey, 2009.

(11) El Halabieh, R. H.; Mermut, O.; Barrett, C. J. Using light to control physical properties of polymers and surfaces with azobenzene chromophores. Pure Appl. Chem. 2004, 76, 1445-1465.

(12) Eastoe, J.; Vesperinas, A. Self-assembly of light-sensitive surfactants. Soft Matter 2005, 1, 338347.

(13) Svetlana, S. Remote control of soft nano-objects by light using azobenzene containing surfactants. J. Phys. D: Appl. Phys. 2018, 51, 013002.

(14) Fomina, N.; Sankaranarayanan, J.; Almutairi, A. Photochemical mechanisms of light-triggered release from nanocarriers. Adv. Drug Deliv. Rev. 2012, 64, 1005-1020.

(15) Leonard, E.; Mangin, F.; Villette, C.; Billamboz, M.; Len, C. Azobenzenes and catalysis. Catal. Sci. Technol. 2016, 6, 379-398.

(16) Holmberg, K.; Jönsson, B.; Kronberg, B.; Lindman, B. Surfactants and polymers in aqueous solution; Wiley Online Library: New York, 2003.

(17) Moroi, Y. Micelles: Theoretical and Applied Aspects; Plenum: New York, 1992.

(18) Merino, E.; Ribagorda, M. Control over molecular motion using the cis-trans photoisomerization of the azo group. Beilstein J. Org. Chem. 2012, 8, 1071-1090.

(19) Hartley, G. S.; Le Fevre, R. J. W. 119. The dipole moments of cis- and trans-azobenzenes and of some related compounds. Journal of the Chemical Society (Resumed) 1939, 531-535.

(20) Tabor, R. F.; Pottage, M. J.; Garvey, C. J.; Wilkinson, B. L. Light-induced structural evolution of photoswitchable carbohydrate-based surfactant micelles. Chem. Commun. 2015, 51, 5509-5512.

(21) Peng, S.; Guo, Q.; Hartley, P. G.; Hughes, T. C. Azobenzene moiety variation directing selfassembly and photoresponsive behavior of azo-surfactants. J. Mater. Chem. C 2014, 2, 8303-8312.

(22) Bandara, H. M. D.; Burdette, S. C. Photoisomerization in different classes of azobenzene. Chem. Soc. Rev. 2012, 41, 1809-1825.

(23) Tan, X.; Zhang, R.; Guo, C.; Cheng, X.; Gao, H.; Liu, F.; Bruckner, J. R.; Giesselmann, F.; Prehm, M.; Tschierske, C. Amphotropic azobenzene derivatives with oligooxyethylene and glycerol based polar groups. J. Mater. Chem. C 2015, 3, 11202-11211.

(24) Tabor, R. F.; Tan, D. D.; Han, S. S.; Young, S. A.; Seeger, Z. L. E.; Pottage, M. J.; Garvey, C. J.; Wilkinson, B. L. Reversible pH-and Photocontrollable Carbohydrate-Based Surfactants. Chem. Eur. J. 2014, 20, 13881-13884.

(25) Richter, M.; Zakrevskyy, Y.; Eisele, M.; Lomadze, N.; Santer, S.; Von Klitzing, R. Effect of pH, comonomer content, and surfactant structure on the swelling behavior of microgel-azobenzenecontaining surfactant complex. Polymer 2014, 55, 6513-6518.

(26) Lee, C. T.; Smith, K. A.; Hatton, T. A. Photoreversible viscosity changes and gelation in mixtures of hydrophobically modified polyelectrolytes and photosensitive surfactants. Macromolecules 2004, 37, 5397-5405.

(27) Schimka, S.; Lomadze, N.; Rabe, M.; Kopyshev, A.; Lehmann, M.; von Klitzing, R.; Rumyantsev, A. M.; Kramarenko, E. Y.; Santer, S. Photosensitive microgels containing azobenzene surfactants of different charges. Phys. Chem. Chem. Phys. 2017, 19, 108-117.

(28) Le Ny, A. L. M.; Lee, C. T. Conformation and dynamics of DNA molecules during photoreversible condensation. Biophys. Chem. 2009, 142, 76-83. 
(29) Zakrevskyy, Y.; Roxlau, J.; Brezesinski, G.; Lomadze, N.; Santer, S. Photosensitive surfactants: Micellization and interaction with DNA. J. Chem. Phys. 2014, 140, 044906.

(30) Diguet, A.; Mani, N. K.; Geoffroy, M.; Sollogoub, M.; Baigl, D. Photosensitive surfactants with various hydrophobic tail lengths for the photocontrol of genomic DNA conformation with improved efficiency. Chem. Eur. J. 2010, 16, 11890-11896.

(31) Chen, S.; Bao, L.; Ou, E.; Peng, C.; Wang, W.; Xu, W. A cationic azobenzene-surfactant-modified graphene hybrid: unique photoresponse and electrochemical behavior. Nanoscale 2015, 7, 1967319686.

(32) McCoy, T. M.; Liu, A. C. Y.; Tabor, R. F. Light-controllable dispersion and recovery of graphenes and carbon nanotubes using a photo-switchable surfactant. Nanoscale 2016, 8, 6969-6974.

(33) Wei, J.; Liu, Y.; Chen, J.; Li, Y.; Yue, Q.; Pan, G.; Yu, Y.; Deng, Y.; Zhao, D. Azobenzene-Derived Surfactants as Phototriggered Recyclable Templates for the Synthesis of Ordered Mesoporous Silica Nanospheres. Adv. Mater. 2014, 26, 1782-1787.

(34) Guo, Y.; Gong, Y.; Gao, Y. a.; Xiao, J.; Wang, T.; Yu, L. Multi-stimuli Responsive Supramolecular Structures Based on Azobenzene Surfactant-Encapsulated Polyoxometalate. Langmuir 2016, 32, 9293-9300.

(35) Hayashita, T.; Kurosawa, T.; Miyata, T.; Tanaka, K.; Igawa, M. Effect of structural variation within cationic azo-surfactant upon photoresponsive function in aqueous solution. Colloid. Polym. Sci. 1994, 272, 1611-1619.

(36) Zakrevskyy, Y.; Titov, E.; Lomadze, N.; Santer, S. Phase diagrams of DNA-photosensitive surfactant complexes: Effect of ionic strength and surfactant structure. J. Chem. Phys. 2014, 141, 164904.

(37) Akamatsu, M.; FitzGerald, P. A.; Shiina, M.; Misono, T.; Tsuchiya, K.; Sakai, K.; Abe, M.; Warr, G. G.; Sakai, H. Micelle Structure in a Photoresponsive Surfactant with and without Solubilized Ethylbenzene from Small-Angle Neutron Scattering. J. Phys. Chem. B 2015, 119, 5904-5910.

(38) Lund, R.; Brun, G.; Chevallier, E.; Narayanan, T.; Tribet, C. Kinetics of Photocontrollable Micelles: Light-Induced Self-Assembly and Disassembly of Azobenzene-Based Surfactants Revealed by TR-

SAXS. Langmuir 2016, 32, 2539-2548.

(39) Israelachvili, J. N.; Mitchell, D. J.; Ninham, B. W. Theory of self-assembly of hydrocarbon amphiphiles into micelles and bilayers. J. Chem. Soc., Faraday Trans. 2 1976, 72, 1525-1568.

(40) Chevallier, E.; Monteux, C.; Lequeux, F.; Tribet, C. Photofoams: Remote Control of Foam Destabilization by Exposure to Light Using an Azobenzene Surfactant. Langmuir 2012, 28, 2308-2312.

(41) Zakrevskyy, Y.; Kopyshev, A.; Lomadze, N.; Morozova, E.; Lysyakova, L.; Kasyanenko, N.; Santer, S. DNA compaction by azobenzene-containing surfactant. Phys. Rev. E 2011, 84, 021909.

(42) Heenan, R. K.; Penfold, J.; King, S. M. SANS at Pulsed Neutron Sources: Present and Future Prospects. J. Appl. Crystallogr. 1997, 30, 1140-1147.

(43) http://www.mantidproject.org.

(44) Wignall, G. D.; Bates, F. S. Absolute calibration of small-angle neutron scattering data. J. Appl. Crystallogr. 1987, 20, 28-40.

(45) Pedersen, J. S. Analysis of small-angle scattering data from colloids and polymer solutions: modeling and least-squares fitting. Adv. Colloid Interface Sci. 1997, 70, 171-210.

(46) Feigin, L. A.; Svergun, D. I. Structure Analysis by Small-Angle X-Ray and Neutron Scattering; Plenum Press: New York, 1987.

(47) http://www.sasview.org/about.html.

(48) Orihara, Y.; Matsumura, A.; Saito, Y.; Ogawa, N.; Saji, T.; Yamaguchi, A.; Sakai, H.; Abe, M. Reversible Release Control of an Oily Substance Using Photoresponsive Micelles. Langmuir 2001, 17, 6072-6076.

(49) Yang, L.; Takisawa, N.; Hayashita, T.; Shirahama, K. Colloid Chemical Characterization of the Photosurfactant 4-Ethylazobenzene 4'-(Oxyethyl)trimethylammonium Bromide. J. Phys. Chem. 1995, 99, 8799-8803.

(50) Gelbart, W. M.; Ben-Shaul, A.; Roux, D. Micelles, membranes, microemulsions, and monolayers; Springer Science \& Business Media: New York, 1994. 
(51) Tanford, C. Micelle shape and size. J. Phys. Chem. 1972, 76, 3020-3024.

(52) Warr, G. G.; Sen, R.; Evans, D. F.; Trend, J. E. Microemulsion formation and phase behavior of dialkydimethylammonium bromide surfactants. J. Phys. Chem. 1988, 92, 774-783.

(53) Dutt, S.; Siril, P. F.; Remita, S. Swollen liquid crystals (SLCs): a versatile template for the synthesis of nano structured materials. RSC Adv. 2017, 7, 5733-5750.

(54) Butt, H.-J.; Graf, K.; Kappl, M. Physics and Chemistry of Interfaces; Wiley-VCH New York, 2004.

(55) Manojlović, J. Ž. The Krafft temperature of surfactant solutions. Therm. Sci. 2012, 16, S631-S640.

(56) Jain, N.; Trabelsi, S.; Guillot, S.; McLoughlin, D.; Langevin, D.; Letellier, P.; Turmine, M. Critical

Aggregation Concentration in Mixed Solutions of Anionic Polyelectrolytes and Cationic Surfactants. Langmuir 2004, 20, 8496-8503.

(57) Billamboz, M.; Mangin, F.; Drillaud, N.; Chevrin-Villette, C.; Banaszak-Léonard, E.; Len, C. Micellar Catalysis Using a Photochromic Surfactant: Application to the Pd-Catalyzed Tsuji-Trost Reaction in Water. J. Org. Chem. 2014, 79, 493-500.

(58) Kano, K.; Tanaka, Y.; Ogawa, T.; Shimomura, M.; Okahata, Y.; Kunitake, T. Photoresponsive membranes. Regulation of membrane properties by photoreversible cis-trans isomerization of azobenzenes. Chem. Lett. 1980, 9, 421-424.

(59) Zemb, T.; Charpin, P. Micellar structure from comparison of X-ray and neutron small-angle scattering. J .Phys. Fr. 1985, 46, 249-256.

(60) Sivia, D. S. Elementary Scattering Theory: For X-ray and Neutron Users; Oxford University Press: New York, 2011.

(61) Bergstrom, L. M.; Grillo, I. Correlation between the geometrical shape and growth behaviour of surfactant micelles investigated with small-angle neutron scattering. Soft Matter 2014, 10, 93629372.

(62) Jackson, A. J. Introduction to Small-Angle Neutron Scattering and Neutron Reflectometry. NIST Center for Neutron Research, Gaithersburg 2008, 12.

(63) Dreiss, C. A. Wormlike micelles: where do we stand? Recent developments, linear rheology and scattering techniques. Soft Matter 2007, 3, 956-970.

(64) Hayter, J. B.; Penfold, J. An analytic structure factor for macroion solutions. Mol. Phys. 1981, 42, 109-118.

(65) Oliver, R. C.; Lipfert, J.; Fox, D. A.; Lo, R. H.; Doniach, S.; Columbus, L. Dependence of micelle size and shape on detergent alkyl chain length and head group. PLoS One 2013, 8, No. e62488.

(66) le Maire, M.; Champeil, P.; Møller, J. V. Interaction of membrane proteins and lipids with solubilizing detergents. Biochim. Biophys. Acta. 2000, 1508, 86-111. 
TABLE OF CONTENTS

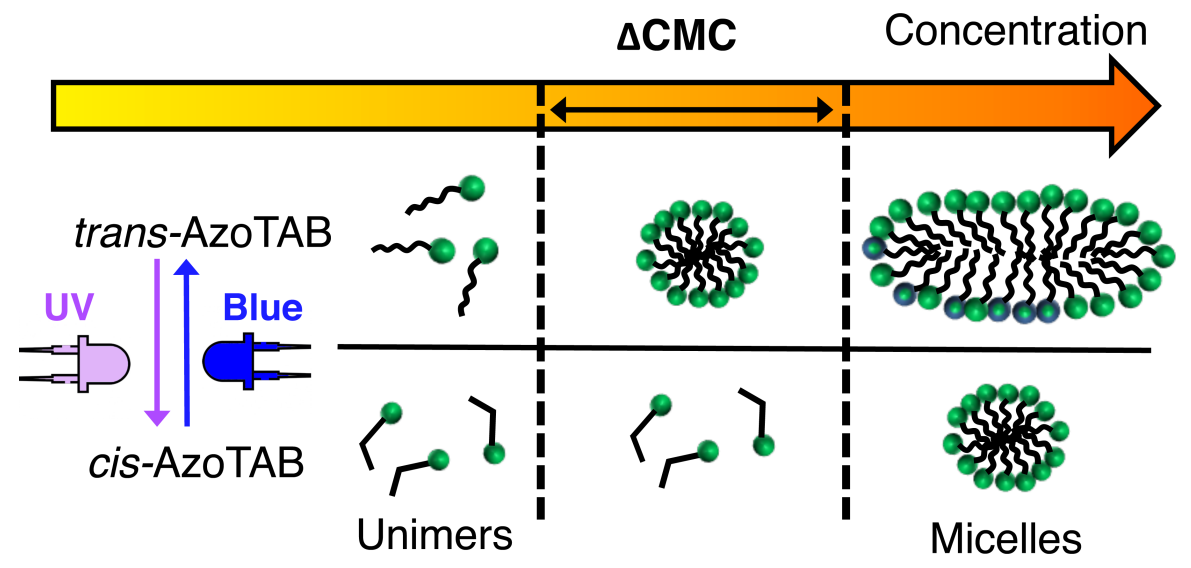

